\title{
Acceptability, Effect and Cost of Mobile-Health on Art Adherence and Assessment of Covid-19 Knowledge Among Youths: A Mixed Methods Sequential Study in Kiryandongo. A Study Protocol Youth Trial
}

Agnes Bwanika Naggirinya ( $\square$ anaggirinya@idi.co.ug )

Makerere University Medical School: Makerere University College of Health Sciences

https://orcid.org/0000-0001-8722-4135

\section{David B Meya}

Makerere University College of Health Sciences

Joseph Rujumba

Makerere University CHS: Makerere University College of Health Sciences

\section{Peter Waiswa}

Makerere University College of Health Sciences

Rosalind Parkes-Ratanshi

Makerere University Infectious Diseases Institute

\section{Research Article}

Keywords: mHealth, Youth, HIV, ART adherence, Barriers, Enablers, cost evaluation This manuscript is written following the SPIRIT checklist and the structure guide by the Directorate of Research and Graduate Training, Makerere University, Kampala Uganda.

Posted Date: December 1st, 2021

DOl: https://doi.org/10.21203/rs.3.rs-892587/v1

License: (c) (1) This work is licensed under a Creative Commons Attribution 4.0 International License. Read Full License 
“Acceptability, Effect and Cost of Mobile-Health on Art Adherence and Assessment of Covid-19 Knowledge Among Youths: A Mixed Methods Sequential Study In Kiryandongo. A Study Protocol Youth Trial”

$\underline{\text { Agnes Bwanika Naggirinya }}^{1,4^{*}}$, David B Meya ${ }^{1,4}$, Joseph Rujumba², Peter Waiswa ${ }^{3}$, Rosalind Parkes-Ratanshi ${ }^{4,5}$

${ }^{1}$ Department of Internal Medicine, School of Medicine, College of Health Sciences, Makerere University, Kampala, Uganda

2 Department of Paediatrics \& Child health, School of Medicine, College of Health Sciences, Makerere University, Kampala, Uganda

${ }^{3}$ Department of Health Policy, Planning and Management, School of Public Health, College of Health Sciences, Makerere University, Kampala, Uganda

${ }^{4}$ Infectious Diseases Institute- College of Health Sciences, Makerere University, Kampala, Uganda.

${ }^{5}$ Department of Public Health \& Primary Care, Institute of Public Health, University of Cambridge, Cambridge, United Kingdom

*Corresponding author: anaggirinya@idi.co.ug

Protocol Version 3.1, Jan 2021

Study ID:

Infectious Diseases Institute -Scientific Review Committee Reference number: 031/2019

School of Medicine, Higher Degrees Research Ethics Committee \#REC Ref: 2019-083

Uganda National Council of Science and Technology Folio Number: HS576ES

Clinical Trial registration: Registered on Jan 2021, Registration number : NCT 04718974, URL

URL: https://ichgcp.net/nl/clinical-trials-registry/NCT04718974

Study Funder: The Academy at Infectious Diseases Institute, College of Health Sciences ,Makerere University

Study Sponsor: Makerere University, Kampala Uganda 


\section{MAKERERE UNIVERSITY}

COLLEGE OF HEALTH SCIENCES

ACCEPTABILITY, EFFECT AND COST OF MOBILE-HEALTH ON ART ADHERENCE

AND ASSESSMENT OF COVID-19 KNOWLEDGE AMONG YOUTHS: A MIXED

METHODS SEQUENTIAL STUDY IN KIRYANDONGO

STUDENT: 2018/HD07/19451U

Dr. Agnes Bwanika Naggirinya

(MBChB, MSc, MMed)

SUPERVISORS

Dr. Rosalind Parkes-Ratanshi (MBBS, MAHons, MRCP, PhD)

Dr. David Meya (MBChB, MMed, CTropMed, PhD)

Dr. Peter Waiswa (MBChB, MPH, PhD)

Dr. Rujumba Joseph (BA. SWSA, MA SSPM, PhD)

A research proposal submitted to Makerere University Directorate of Research and Graduate Training in partial fulfilment for the award of a Doctor of Philosophy degree of Makerere University

CJuly 2020 
SUPERVISORS SIGNATURE PAGE

1. Dr. Rosalind Parkes-Ratanshi (MBBS, MA Hons, MRCP, PhD)

2. Dr. David Meya (MBChB, MMed, C Trop Med, PhD)

3. Dr. Peter Waiswa (MBChB, MPH, PhD)

4. Dr. Joseph Rujumba (BA. SWSA, MA SSPM, PhD) 


\section{Contents}

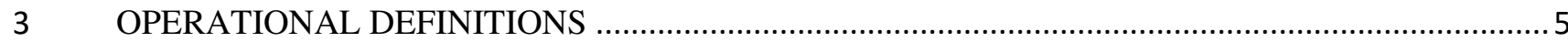

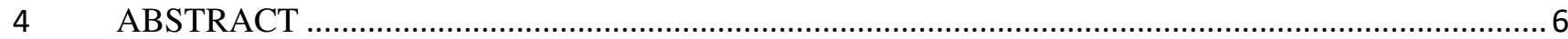

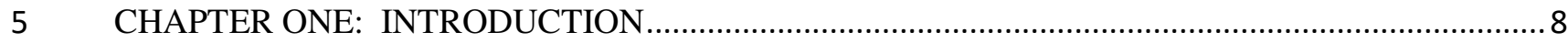

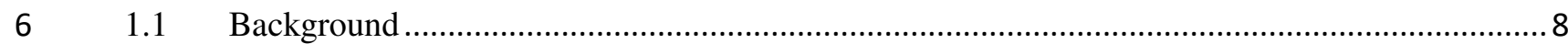

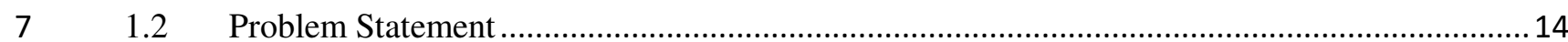

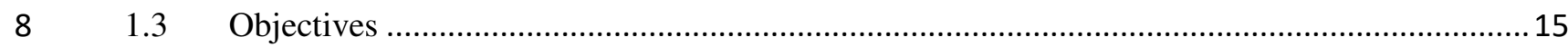

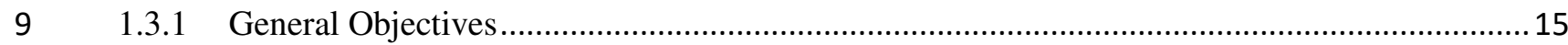

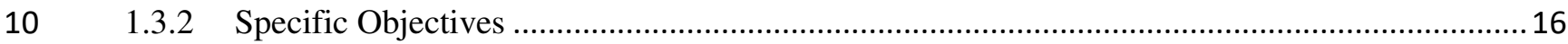

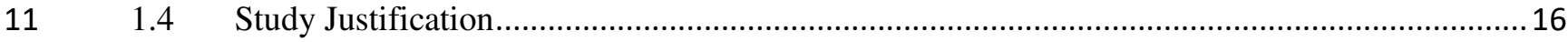

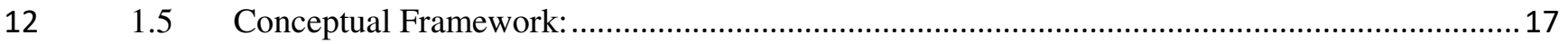

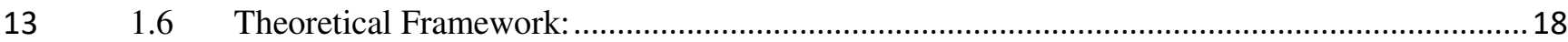

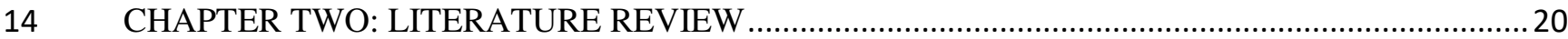

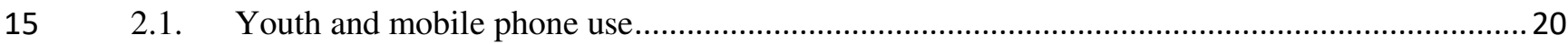

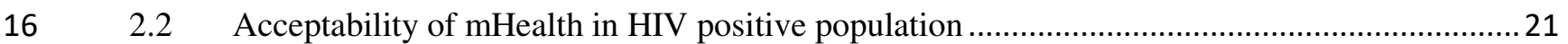

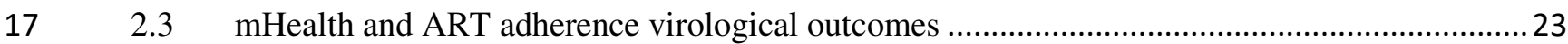

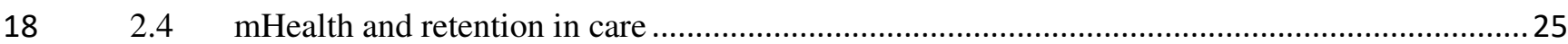

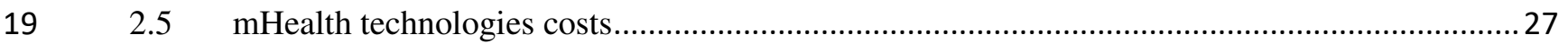

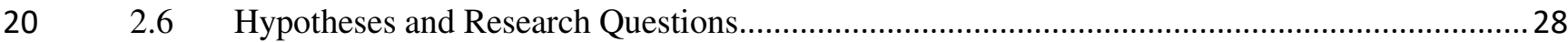

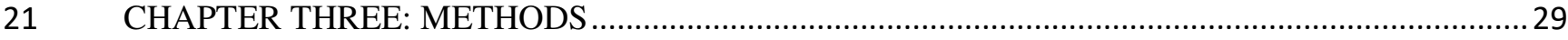

Page 3 of 76 


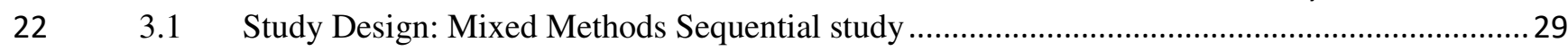

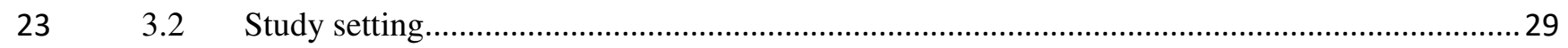

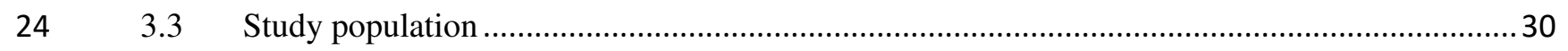

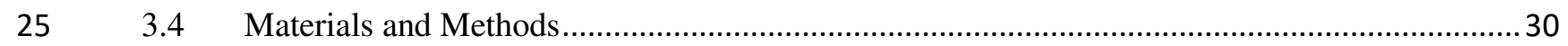

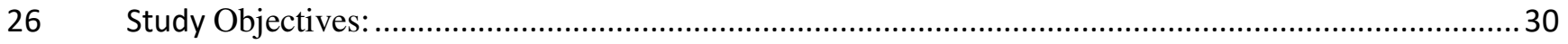

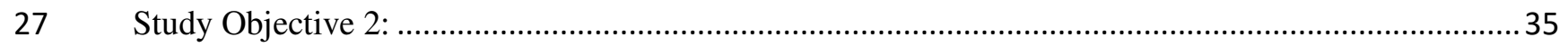

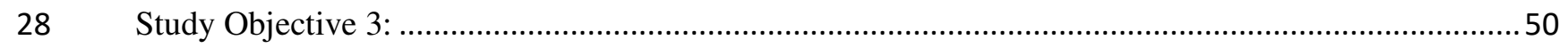

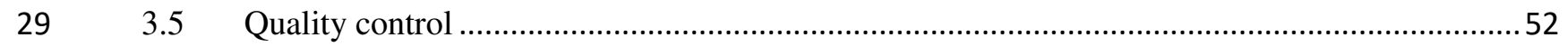

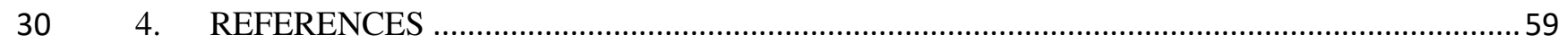




\section{OPERATIONAL DEFINITIONS}

38 Adherence to ART: Self- reported adherence to ART as depicted on the mHealth dash board or

39 HIV card

40 Loss to follow-up: Missing 2 visits consecutively, not able to ascertain vitals

41 mHealth: use of mobile technology e.g. mobile phones and other wireless technology for health-

42 related activities

43 Retention in care: Continuous engagement in clinical care for 1 year

44 Virological suppression: Viral load less than 1000 copies/ml

45 Youth Living with HIV/AIDS: Persons living with human immunodeficiency virus/ acquired 46 immunodeficiency syndrome aged 15 to 24yrs (United Nations General Assembly Resolution

47 A/RES/50/81 ("World Programme of Action for Youth to the Year 200 and Beyond") 48 
51 Background: AIDS is the leading cause of death among young people in sub-Saharan Africa.

52 Adherence to antiretroviral therapy is the principal determinant for achieving and sustaining viral

53 suppression, which decreases progression to AIDS and reduces risk of mortality.

54 Few studies have evaluated mHealth adherence tools among youths in resource-limited settings.

55 We aim to evaluate whether mHealth tool improves ART adherence outcomes among youth

56 receiving ART at a rural district in Western Uganda.

57 The Corona virus disease outbreak was announced a Public Health Emergency of International

58 concern on Jan 2020; and declared a global pandemic by World Health Organization on Mar,

59 2020. In rural areas, there is little data on knowledge and myths on COVID among youths.

60 General objective: To assess acceptability, effect and cost of the mHealth tool on ART

61 adherence, knowledge and myths on COVID-19 among youth initiating and on ART at

62 Kiryandongo District.

63 Methods: This is a mixed method sequential exploratory study, with the qualitative study

64 conducted first followed by a randomized control trial and healthcare cost evaluation. The

65 qualitative study will assess barriers, enablers of adherence and acceptability of mHealth among

66 youths receiving care at three health facilities in Kiryandongo District. The randomized control

67 trial of 206 youth initiating ART to either Standard of Care or mHealth tool plus Standard of care

68 to assess effect of mHealth tool on ART adherence and retention in care. Through a basic cell

69 phone, participants in the intervention arm will receive pill reminders, clinic appointment

70 reminders, health voice messages and self-reported symptoms in addition to standard of care.

71 Collection of data on knowledge and myths on COVID-19, HIV and sexual behavior.

72 The health care evaluation nested within randomized trial, will assess the cost of intervention in 73 comparison to Standard of care. 
Version 3.1, Jan 2021

74 Discussion: This project will determine acceptability, effectiveness of mHealth, knowledge \&

75 myths on COVID-19 and cost of delivering pill and clinic appointment reminders, and voice

76 messages to a population with suboptimal ART adherence in a resource-limited setting.

77 Trial registration: Fully registered under clinicaltrials.gov by $20^{\text {th }}$ Jan 2021 . The study is

78 ongoing. Recruitment started Aug 2020.

79 Clinical Trial registration: NCT 04718974

80 https://ichgcp.net/nl/clinical-trials-registry/NCT04718974

81 Key words: mHealth, Youth, HIV, ART adherence, Barriers, Enablers, cost evaluation

82 This manuscript is written following the SPIRIT checklist and the structure guide by the

83 Directorate of Research and Graduate Training, Makerere University, Kampala Uganda..

84

85

86

87

88

89

90

91

92

93

94 
CHAPTER ONE: INTRODUCTION

98

99

100

101

102

103

104

105

106

107

108

109

110

111

112

113

114

115

116

\subsection{Background}

Human Immunodeficiency Virus (HIV) continues to be a global public health threat. Globally, about 37.9 million [32.7-44.0 million] people were living with HIV (PLWH) in 2018 of whom 25.7 million were in sub-Saharan Africa (1). An estimated 3.9 million[2.1-5.7 million] youth were living with HIV (YLWH) globally in 2017, most of whom live in sub-Saharan Africa $(2,3)$. Every week in 2018, 6,200 young women aged 15-24 years became infected with HIV around the world (4). In sub-Saharan Africa, $80 \%$ of new HIV infections, among young people aged 15-24, occur in girls. Young women (15-24 years) are twice as likely to be living with HIV as young men (5).Young people are the only population group in which AIDS-related deaths are increasing (6). AIDS is the leading cause of death among young people in sub-Saharan Africa (2).

In Uganda, there were 160,000 YLWH in 2018 (4). An estimated 53,000 new HIV infections occurred among youth in 2018 , accounting for $26 \%$ of HIV incidence in the country (2). Uganda is implementing the Joint United Nations Programme on HIV and AIDS (UNAIDS) 90-90-90 targets, which aim to achieve $90 \%$ of PLWH knowing their status, $90 \%$ of those who know their status accessing antiretroviral therapy (ART) and 90\% of people on ART achieving virologic suppression by 2020 (7). With regard to attaining global 90-90-90 targets, this means that $81 \%$ of people with HIV globally receive ART and $73 \%$ of them are virally suppressed. In 2018 in Uganda, $84 \%$ of people with HIV knew their status, $72 \%$ of PLWH were on ART and 64\% of PLWH were virally suppressed $[(2,4)$. Viral suppression rates were much lower among youth in Uganda in 
Version 3.1, Jan 2021

117 2017: 44.9\% for females and 32.5\% in males (8). Young people (15-19 years) in Uganda are more 118 likely to drop out of HIV care than children or adults because of fear of disclosure, stigma and 119 discrimination (9)

120 The Infectious Diseases Institute (IDI) is an implementing partner for the President's 121 Emergency Plan for AIDS Relief (PEPFAR) in three of Uganda's seven regions, and provides care 122 for approximately one-in-three of the 1,167,107 Ugandans receiving ART. In 2017, 18 districts 123 supported by the IDI had viral suppression rates between $38 \%$ and $87 \%$. Kiryandongo Hospital, 124 one of the HIV care facilities supported by the IDI, was chosen as the study site because it had a 125 total of 1,322 youth (15-24 years) registered in care by June 2019. The number of YLWH accessing 126 care at this hospital has increased over the years, from 1,107 in 2017 to 1,308 in 2018. In 2018, a 127 total of 637 new youth were registered for care at the unit, and by Jun 2019, an additional 218 128 youths had been registered into care. Overall retention in care at the hospital was $76 \%$ in 2017 and 129 improved to $79 \%$ in 2018. The viral suppression rate was $72 \%$ and $79 \%$ in 2017 and 2018 , 130 respectively, but this is still below the national and UNAIDS target of $90 \%$.

131 The World Health Organization (WHO) defines mobile health (mHealth) as "medical or 132 public health practice supported by mobile devices" including mobile phones (10). The WHO 133 recommends mHealth interventions to support HIV adherence. Prior mHealth research studies 134 have shown improved virological outcomes, decreased risk of non-adherence, good retention in 135 care, strengthened health systems through ART adherence and clinic reminder support and 136 improved self-reported adherence and viral load suppression among non-adherent youth (11) (12) 137 (13, 14) (Rebecca Schnall, M.R.,2010). The Call for Life Study (CFL) was an mHealth 138 intervention trial which aimed to improve outcomes in HIV patients using mobile phone based 139 interactive software support (ClinicalTrials.gov NCT 02953080). Between August 2016 and 
140 November 2018, 1,031 PLHIV were screened and 600 participants enrolled (300 per site). They

141 were randomised in a 1:1 ratio, and followed at an urban site (Infectious Diseases Institute Clinic)

142 and a peri-urban HIV clinic (Kasangati Health Centre) to either Standard of Care (SoC) face-to-

143 face counsellor adherence support or SoC plus the Call for Life Uganda (CFLU) mHealth tool.

144 CFLU used Interactive Voice Response (IVR) or text messages delivered to participants via

145 MOTECH-based Connect for Life ${ }^{\mathrm{TM}}$ (Janssen, Global Public Health-J\&J). The CFLU tool offered

146 daily pill reminders, appointment visit reminders, symptom reporting and management and weekly

147 health information tips. The primary objective of the study was to assess quality of life utilizing

148 medical outcome study scores (MOS) and physical health scores (PHS) in people living with HIV

149 (PLWHIV) accessing mobile phone-based support using the CFLU tool. Despite having no

150 statistically significant observed difference in mean percentage scores of MOS-HIV, Mental

151 Health Scores and PHS at baseline and 6 months between CFL and SoC arms, a 5-point

152 improvement in PHS favoring the intervention group was noted among participants starting first

153 line ART or switching to second line (ANCOVA 4.01; $\mathrm{p}=0.048)$ (15).

154 Adherence to ART is the principal determinant for achieving and sustaining viral suppression,

155 which decreases progression to AIDS and reduces risk of mortality (16). Suboptimal adherence 156 includes missed or late doses, sub-therapeutic dosing and treatment interruptions or 157 discontinuations (17) (18). Sustaining adherence to ART among youth is challenging perhaps 158 because of treatment fatigue (for those congenitally infected), side effects, drug interactions, drug 159 toxicities, sub-optimal regimens, viral fitness, malabsorption, incomplete emotional and 160 psychological development, lifestyle choices and lack of dedicated health services (19). Sub161 optimal adherence leads to sub-therapeutic plasma concentrations of antiretroviral drugs which 162 may speed up the development of drug resistance, limit future drug options and permit 
Version 3.1, Jan 2021

163

164

165

166

167

168

169

170

171

172

173

174

175

176

177

178

179

180

181

182

183

184

185 transmission of resistant strains (20). These factors highlight the difficulty of maintaining high adherence among youth.

HIV services oriented toward adult care are perceived by youth as poor quality and intimidating which discourages retention in care (21). Thus, youth are more likely to drop out of HIV care than adults (22) (23). There is urgent need to develop interventions to improve ART adherence among youth. Few studies have evaluated mHealth adherence tools among youths in resource-limited settings. This study aims to evaluate whether the CFLU mHealth tool improves ART adherence outcomes among youth receiving ART at a rural district_in Western Uganda.

When initiated on ART, a high level of sustained adherence is necessary to suppress viral replication and improve immunological and clinical outcomes, decrease risk of developing ARV drug resistance and reduce the risk of HIV transmission (24). The proportion of youth with viral load suppression in Uganda is suboptimal; only $44.9 \%$ of females and $32.5 \%$ of males achieved viral suppression in 2017. Effective implementation of evidence-based interventions is needed if the last two 90s of the UNAIDS 90-90-90 targets are to be achieved.

Previous work has shown that mHealth interventions improve virological outcomes (25) (26), decrease risk of non-adherence (27), improve retention in care (28), strengthen health systems through ART adherence and clinic reminder support (29) and improve self-reported adherence and viral load suppression among non-adherent youth (30). The Call for Life Uganda mHealth tool was developed by Janssen Global Public Health Research and Development, in close collaboration with the Infectious Diseases Institute (IDI), and tailored it to the needs of PLHIV in Uganda. Call for Life Uganda (CFLU) is based on the CONNECT FOR LIFE ${ }^{\mathrm{TM}}$ technology and the MOTECH platform. CONNECT FOR LIFE ${ }^{\mathrm{TM}}$ is an m-health technology initially developed as a community information technology platform for health initiatives to improve care in patients in developing 
Version 3.1, Jan 2021

countries. The platform interacts with patients using basic mobile phone technology and with healthcare providers through a web-based interface. This Interactive Voice Response (IVR) software was first piloted by Janssen and the Grameen Foundation in India, and was called “Treatment advice by Mobile Alerts”(TAMA) to support HIV patients in India.

The Call for Life Uganda (CFLU) study randomized 600 PLHIV to receive enhanced adherence support with daily SMS or Interactive Voice Response (IVR) call reminders, standardized health education messages and facility based standard of care (intervention arm) or facility standard of care only (SOC) in a 1:1 ratio (ClinicalTrials.gov NCT 02953080). A total of 331 health education messages were designed and developed by IDI and Straight Talk FoundationUganda staff, using National HIV prevention guidelines. These messages were piloted with expert patients at the Infectious Diseases Clinic (IDC) at Mulago Hospital in Kampala, Uganda, translated into three local languages (Luganda, Kiswahili and Runyakitara) and relayed to patients using the platform once a week or on request during the daily pill reminder call. Patients could also call in using a toll-free number to request health education messages, or report any symptoms related to the disease, drug or any concomitant illness. The CFLU study participants were prospectively followed 6 monthly for 24 months. The study's main outcome was to assess quality of life utilizing Medical Outcome Study Scores-HIV (MOS-HIV), Mental Health Scores (MHS) and Physical Health Scores (PHS) in PLWHIV accessing mobile phone-based adherence using the CFLU tool. There was no statistically significant observed difference in mean percentage score of MOS-HIV, MHS and PHS at baseline and 6 months between CFLU and SoC arms. In those starting first line ART or switching to second line, there was a significant improvement in PHS (ANACOVA 4.01, $\mathrm{p}=0.048$ ) of 5-points difference or greater. Qualitative data suggested high acceptance and positive 
Version 3.1, Jan 2021

experiences with the CFLU tool in PLHIV. Nearly all participants (98\%) selected interactive voice response over text messages $(15,31)$.

IVR is a useful and flexible mHealth tool which can be used for computer-assisted screening of a population (32) or in-depth daily assessments of risk behaviours $(33,34)$. It can also be used as an external aid to promote participant medication adherence $(35,36)$ or used as a medium to relay health messages or advice intended to improve health behaviours(32). IVR may be programmed to receive incoming calls from participants, place automated outgoing calls, or a combination of both (37). Using this strategy in CFLU, this mHealth tool is able to track study participants, allows investigators to receive data nearly in real-time and to intervene should a problem occur with the study participant, before it is too late. The Call for Life System has functionality to support individualised pill reminders, visit reminders, health message calls and self-reported symptoms and management. mHealth apps have been developed for asthma, chronic obstructive pulmonary disease, diabetes, malaria, tuberculosis, and HIV as well as for specific populations, e.g., mother and baby (38). Call for Life System can be adopted for use in Ugandan YLWH to aid in ART and clinic adherence, knowledge sharing and symptom management. Coronavirus disease (COVID-19), a viral infection with high infectivity and humanhuman transmissibility, is caused by severe acute respiratory syndrome coronavirus 2 (SARSCoV-2). The SARS-CoV-2 is probably of zoonotic potential, due to the allegations that the virus was transferred to people at a food market that trades in wildlife in Wuhan, China (39).

The state of lock-down due to COVID-19 in many parts of the world has led to the halting of services (40)and Uganda health service provision has not been spared either. Uganda got first country wide lockdown on 22nd Mar 2020, this lockdown disrupted treatment continuity and globally the supply chains were halted and most patients in chronic care faced challenges (40). 
Version 3.1, Jan 2021

231 There is moderate awareness and knowledge of COVID-19 among educated population in

232 India(40), but little is document among youths in rural areas, literature search revealed one

233 documentation on knowledge, attitude and practices of HCW in Makerere University teaching

234 hospital(41). A poor understanding of the disease among youths can result in delayed identification

235 and treatment leading to rapid spread of infections.

236

237

238

239

240

241

242

243

244

245

246 247 death for young women aged 15-24 years in Africa (43).

248

249

250

251

252

\subsection{Problem Statement} occur among youth 15-24 years (UNAIDS).

About 5 million[ 2.1-5.7 million] youth were living with HIV (YLWH) globally in 2018, most of whom live in sub-Saharan Africa $(2,3)$ (https://www.who.int>adolescents). AIDS is the leading cause of death in Africa (https:// www.avert.org). Globally, 30\% of all new HIV infections

Youths living with HIV (YLWH) have low viral load suppression rates due to poor ART adherence compared with older adults. According to the UPHIA 2017 report, a household-based national survey conducted in Uganda from August 2016 to March 2017, approximately 55\% of

young females and $68 \%$ of young males had unsuppressed HIV viral load (42). Persistent viremia is associated with development of drug resistance, low immunity which increases the risk of opportunistic infections and death (20). AIDS-related illnesses remain the second leading cause of

Improved linkage and retention in care coupled with improved adherence for viral suppression are paramount in contributing to the UNAIDS second and third 90s and improving the HIV treatment continuum (44). Poor viral load suppression is mainly due to non-compliance to clinic visits, lack of knowledge on ART, inconsistent swallowing of pills and poor pill adherence (45). We hypothesize that early follow-up of missed appointments coupled with adherence support 
Version 3.1, Jan 2021

253 for the first 6 to 12 months of initiating ART could overcome the current problem faced by youth.

254 Existing evidence indicates that Intensive Adherence Counselling (IAC) for those who are not 255 suppressed may not be effective. A retrospective chart review demonstrated that suppression rates 256 were low among ART-treated children with virological failure that completed the three 257 recommended IAC sessions (46).

258 Mobile health interventions have been effective in improving ART adherence and viral 259 suppression is a proxy measure for the adherence. A study of SMS text messaging in Italy found 260 that the proportion with undetectable HIV RNA viral load increased from $42.3 \%$ at baseline to $26176.8 \%, 71.5 \%, 76.2 \%$ at months 3, 6, and 9, respectively $(\mathrm{P}=0.001)$ (47). In rural Uganda, text 262 messaging was effective in increasing ART adherence and the authors recommended that it be 263 explored in youth $(13,48)$.

264 Poor ART adherence due to missed clinic visits and lack of drug refills, forgetfulness, stigma 265 comorbid conditions and psychosocial issues are the leading causes of poor viral suppression in 266 youth. Current use of Intensive Adherence Counselling for non-adherent, non-suppressed patients

267 has not been shown to be effective, and there is still a gap in providing daily pill reminders, visit 268 reminders, HIV and ART knowledge and adherence support. All this can be achieved through use 269 of interactive voice response tool (IVR), which this study will provide through the Call for Life 270 tool.

$271 \quad 1.3$ Objectives

\section{$272 \quad$ 1.3.1 General Objectives}

273 To assess acceptability, cost and effect of mHealth tool on ART adherence and knowledge of

274 COVID-19 among youths initiating ART in Kiryandongo. 
276 I. To assess barriers, enablers of adherence for ART among Youth in Kiryandongo at 277 baseline and study end.

278 II. To assess acceptability of mHealth for ART adherence among Youth in Kiryandongo at $279 \quad$ baseline and study end

280 III. To evaluate effect of the mHealth CFLU tool on ART adherence at months 6 and 12

281 IV. To evaluate the cost of the mHealth CFLU tool in comparison to standard of care at 12 282 months

$283 \mathrm{~V}$. To assess knowledge of COVID-19 among youths in rural setting

\section{$284 \quad 1.4 \quad$ Study Justification}

Over $55 \%$ of YLWH in Uganda are not virologically suppressed. Patient-centered 286 approaches exploiting mHealth have been shown to improve adherence to ART and viral 287 suppression. Due to poor clinic attendance by youth, Intensive Adherence Counselling (IAC) alone 288 cannot help suppress detectable viral load in this group. Evidence-based interventions are needed 289 to complement standard of care adherence counseling. The 2013 WHO consolidated guidelines on 290 the use of antiretroviral drugs for treating and preventing HIV infection, suggested use of 291 automated Short Message System (SMS) reminders of scheduled clinic visit to improve clinic 292 attendance (49). Experience with the recently concluded mHealth study (Call for Life Study) at 293 IDI shows that $98 \%$ of the study participants preferred voice calls instead of text messages. 294 Participants cited confidentiality, voice tone and privacy related to voice calls as reasons for this 295 preference. CFLU is an open source mobile-phone based software designed to send short 296 messaging services (SMS) and Interactive Voice response (IVR) calls. Additional services include 
297 pill reminder support, adherence support, visit appointment reminders, symptom report 298 management and health information tips (FIGURE1). However, this mHealth intervention has not 299 been implemented among the YLWH in Uganda.

It is estimated that $53.2 \%$ of Ugandans have access to mobile phones; 23.2 million 301 Ugandans were mobile phone subscribers in 2016 (50). According to the Pew Research Centre, 302 Ugandan youth are more likely to use mobile phones than adults (51). This study found that $66 \%$ 303 and $51 \%$ youth had ever used text messages or sent/viewed videos or pictures, respectively, 304 highlighting their ease and comfort with cell phone use. In a systematic review of 25 mHealth 305 programs targeting youth sexual reproductive health (SRH) found that most (67\%) were conducted 306 in Africa. Nearly all $(87 \%)$ used mHealth as a health promotion tool to facilitate knowledge and 307 behavior change. A minority (18\%) used mHealth to link users to essential SRH services including 308 family planning counselling services, medical abortion, post-abortion care and HIV treatment. 309 Evidence is emerging that mobile phones are an effective way to reach youth and to achieve 310 knowledge and behavior change (52).

311 Few studies have evaluated mHealth adherence tools among youths in resource-limited 312 settings. This study aims to evaluate whether the CFLU mHealth tool improves ART adherence 313 outcomes among youth receiving ART at a rural hospital in Western Uganda.

314 This study will join Uganda in implementing the Joint United Nations Programme on HIV and 315 AIDS (UNAIDS) 90-90-90 targets, which aim to achieve 90\% of PLWH knowing their status, $31690 \%$ of those who know their status accessing antiretroviral therapy (ART) and 90\% of people on 317 ART achieving virologic suppression by 2020 (7).

$318 \quad 1.5$ Conceptual Framework: 
Version 3.1, Jan 2021

320 Conceptual framework showing different factors which affect antiretroviral therapy (ART)

321 adherence and resultant viral suppression

$322 \quad 1.6 \quad$ Theoretical Framework:

323 Theoretical framework, is based on the Information-Motivation-Behavioral Skills Model (53)).

324 IMB model commonly utilized in HIV to understand behaviors, in this study, we plan to provide

325 information to the youth, motivate through use of mHealth tools in order to impact behavior 326 change and improve pill adherence (Figure 3).

The IMB Model, (54) was developed to explain HIV risk and preventive behavior. The model recognizes three key constructs needed to engage in a given health behavior: information,

329 motivation and behavioral skills. Information involves the provision of correct information and 330 dismissing of incorrect information. Motivation refers to both personal and social motivation 331 (perceived social support for engaging in certain behavior) and behavioral skills refer to perceived 332 self-efficacy for carrying out a certain behavior (55).

Based on the IMB model (56), existing information affecting adherence will be elicited 334 first, and this information may be around social-psychological factors, anthropological factors, 335 stigma and HIV/ART knowledge among youth. Most of the information regarding adherence of 336 ART in this group will be assessed at baseline and study end during the qualitative data collection, 337 stigma will be prospectively collected under the second objective. 339 improved knowledge in sexual reproductive health and facilitated behavior change (52). The IMB 340 model posits that provision of information and motivation influences behaviours. We hypothesize 341 that the CFLU tool will address existing knowledge gaps among YLWH through provision of 
Version 3.1, Jan 2021

342 health messages about HIV, ART and medication adherence. Daily pill reminders and visit

343 reminders will motivate youth and will help to address forgetfulness and busy schedules, factors

344 which were the main causes of medication non-adherence and missed visits in the Call for Life

345 study. Addressing these factors will influence adherence behaviours.

346

Prior work suggests that patients with higher HIV knowledge are twice as likely to keep

347 their appointments as those who are less knowledgeable. Knowledge of HIV coupled with a good 348 client-provider was associated with increased CD4 count and a five-fold likelihood of achieving 349 undetectable VL (57). Lack of knowledge of correct dosing and time to take drugs was associated 350 with non-adherence in those starting ART (58). HIV knowledge has also been shown to be 351 associated with improved quality of life (59).

352 Lastly, evaluation of the effect mHealth tool will include assessment of stigma scores at 353 baseline and follow-up, HIV knowledge at baseline and follow-up, and adherence as measured by 354 clinic attendance (visit adherence), phone interaction and viral suppression.

355 The IMB model was chosen because the intervention will provide information through health tip 356 messages and motivation through daily calls. According to prior recipients of the CFLU mHealth 357 intervention, voice calls and voice tones encouraged and motivated them to continue taking 358 medication, which impacted their behaviours including honoring clinic visit appointments, taking 359 medication on time and willingness to report any symptoms they may have had. These factors may 360 have improved clinic attendance, ART adherence and viral suppression. This model is a better fit 361 for the CFLU mHealth tool than similar behavior models e.g. the Capability-Opportunity362 Motivation-Behavioral (COM-B) model. 


\subsection{Youth and mobile phone use}

366 The use of mobile phones and other mobile technologies for improving health through research and practice is growing quickly, particularly in areas with difficult-to-reach populations or where

368 the healthcare infrastructure is less developed (38). In the Kampala Youth Survey 2014, it was 369 noted that nearly half of the youth owned and used phones daily (60). The study assessed 370 psychosocial characteristics based on phone ownership and use and found that those who initially 371 owned and used mobile phones were educated, young, wealthy, male and living in urban areas 372 (60). More recently, cell phone use has expanded to include those living in rural areas, the elderly, 373 and those with less resources to an estimated 23 million subscribers in Uganda (61). In the prior 374 Kampala Youth Survey (2011), 47\% of youth reported owning a mobile phone. Ownership did not 375 vary by sex but was more common among youth above 18 years of age. Mobile phone ownership 376 was more common among those who reported taking care of themselves at night, who reported 377 current drug use and who reported trading sex for money, food or other goods.

As the burden of HIV continues to grow among adolescents and youth, mHealth 379 technology could address many of the healthcare needs of YLWH including adherence to HIV 380 medications, retention in care and self-management (62), which are critical needs for this priority 381 population. mHealth technology could bridge the divide in healthcare delivery in underserved 382 minority groups. Since ownership of a mobile device is common among the youth, use of mHealth 383 can help increase engagement in HIV care (52). Due to the high incidence of HIV among 384 minorities, adolescents and underserved youth, and their reliance on mobile technology, it is 
Version 3.1, Jan 2021

385 imperative to develop health information technology (HIT) tools tailored to the needs of this 386 population $(63,64)(12)$.

387 Overall HIV incidence in Uganda has been declining since 2010. However, the new HIV infections 388 are disproportionately concentrated among young people. Given high penetration of mobile phone 389 technology, there is growing support for using mobile health (mHealth) programs to reach 390 vulnerable populations $(65,66)$. Given that nearly half of youth in Uganda own or use mobile 391 phones daily, new research is needed to determine next steps for mobile health (mHealth), 392 including the feasibility of using mobile phones for data collection and interventions with this 393 hard-to-reach population (Swahn et al, Aug 2014).

\subsection{Acceptability of mHealth in HIV positive population}

Several studies on the acceptability and feasibility of mHealth in PLWH have been done, but

396 few of these were conducted among YLWH. One study in South Africa examined short message 397 service (SMS) and/or community-based directly observed antiretroviral therapy (cDOT) as 398 interventions to improve ART adherence for preventing mother- to-child HIV transmission. This 399 study showed that SMS and or cDOT are feasible in this setting. However, safe HIV status 400 disclosure to treatment supporters and confidentiality of text messaging content about HIV and 401 ART were deemed to be crucial implementation factors (67).

$402 \quad$ Few mHealth studies have been conducted among youths despite several studies of mHealth 403 apps $(68,69)$, usability of the devices (70), and negative effects of sexualized video apps (71). 404 Brown et al, conducted 6 focus group discussions to explore adolescent use of mobile technology 405 for meeting their health information needs in Columbia, when given smart phones with mHealth 406 apps. The study assessed health seeking information behavior among adolescents and found that 
Version 3.1, Jan 2021

407 the main motivation for using mHealth app was their performance speed and information needs.

408 Barriers for use of the app included use of non-phone app technology (i.e. phone, books), non-

409 mobile resources (i.e. parents, teachers, siblings), and other health related entities not directly

410 related to the usability of mHealth (72) (73).

411 Breach of confidentiality may occur following receipt of text messages. In an mHealth study

412 among diabetics in Ethiopia, willingness to receive text messages for appointment reminders was

$41370.5 \%,(74)$. In North West Ethiopia, willingness to receive text message medication reminders

414 among PLWH was only 50.9\%, perhaps because use of SMS can't guarantee privacy and

415 participants may have felt it was not safe to receive the mHealth support (75). In the rural United

416 States, a survey carried out among 24 respondents, showed a demand for mHealth services, out of

417 the 24 respondents, $63 \%$ were in good health, a total of $65 \%$ were willing to receive prerecorded

418 messages for appointment reminders from the doctor (76). In rural India, willingness to receive

419 mobile phone reminders for drug adherence was high (98\%) among the 479 respondents. Of these,

$420424(89 \%)$ preferred voice calls alone to other forms of communication (77).

421 In a rural Ugandan setting, a cross-sectional study found that over $60 \%$ of patients could

422 potentially benefit from a mobile phone-based ART adherence support (78). Most of the mHealth

423 studies conducted are SMS studies and only one used IVR. A qualitative study among youth

424 assessed SMS based intervention to improve ART adherence in 2 clinics in Kampala, Uganda.

425 Results suggested improved adherence could be achieved through reminders and social support.

426 However, youth also suggested "intervention logistics related to content, frequency, timing and

427 two-way messages" would be helpful to practitioners in the field (79). Another qualitative study

428 in South-Western Uganda nested within sixty-two pilot intervention study participants found that

429 SMS reminders and real-time adherence monitoring led to the habit of adherence, and real-time 
Version 3.1, Jan 2021

monitoring was interpreted as "being seen", "being cared about" which transformed their moods (80). In rural Uganda cohort of 276 participants on ART, more than half (64\%) possessed a mobile phone and were willing to be contacted after a missed clinic appointment. In $79 \%$ of episodes of missed visits, patients presented for refills within a mean duration of 2.2 days $(\mathrm{SD}=1.2$ days) after mobile phone call reminder (81). Another cross-sectional survey among PLWH in rural Uganda assessed the "acceptability of and preferences for text messaging, preferences for laboratory results notification, privacy and confidentiality and cell phone use and literacy". All the participants expressed interest in the service although they had challenges regarding "interpreting messages, discouragement upon learning bad news, and technical issues" (82). In a feasibility, validity and acceptability study of real-time adherence monitoring using a Wise pill wireless electronic adherence monitor (EAM) and self-reported missed doses via interactive voice response (IVR) and short message service (SMS), among 49 adults and 46 children in Mbarara, Uganda, adherence was higher in children than adults $(92.8 \%$ vs $89.5 \%)$ by EAM, and $99-100 \%$ for both adults and children by IVR/SMS self-report (83).

\section{3 mHealth and ART adherence virological outcomes}

Poor adherence to antiretroviral treatment is a public health challenge associated with development of drug resistant strains necessitating initiation of costly second line therapy, and possibility of transmission of drug-resistant HIV by the non-adherent spouse (84-86).

A quasi-experimental cohort study involving $150 \mathrm{HIV}$-infected individuals from Bangalore, India, showed that among participants on antiretroviral therapy (ART) between April and July 2010, optimal adherence increased from $85 \%$ to $91 \%$ patients during the intervention period, an effect that was maintained 6 months after the intervention was discontinued $(\mathrm{p}=0.016)$. Both, IVR 
452 calls and SMS reminders were considered nonintrusive and not a threat to privacy. A significantly

453 higher proportion agreed that the IVR was helpful compared to the SMS (p<0.001) (87).

454 A systematic review done by Ridgeway $\mathrm{K}$ et al, of the peer-reviewed and grey literature 455 published between 2010 and 2015 to identify interventions designed to improve antiretroviral 456 adherence among adults and adolescents in low and middle-income countries, found 43 457 interventions for adults, 6 involved both adults and adolescents and only 2 were among adolescents 458 (88). Two systematic reviews $(89,90)$ found modest evidence for the benefits of mHealth 459 technology. Both reviews recommended mHealth implementation, but argued that high quality 460 (and adequately powered) clinical trials that measure clinical outcomes are essential (91).

461 Garofalo et al, May 2016, conducted a randomized trial of a two-way, personalized daily text 462 messaging intervention to improve adherence to antiretroviral therapy (ART) among 105 poorly 463 adherent HIV-positive adolescents and youth, ages 16-29. The average effect estimate over the 6464 month intervention period was significant for $\geq 90 \%$ adherence $(\mathrm{OR}=2.12,95 \% \mathrm{CI}=1.01-4.45$, $465 \mathrm{p}<.05)$ and maintained at 12-months (6-months post-intervention) (30).

467 shown to improve adherence to cART and promote achievement of suppressed HIV plasma viral 468 loads(92, 93). A systematic review by H. Anglada-Martine et al, revealed that the use of text 469 messages or mobile applications to enhance adherence to medication do seem to have been 470 beneficial, as $65 \%$ of the studies evaluated had positive outcomes. However, they recommended 471 that more high-quality studies be conducted in order to demonstrate whether this type of 472 technology reduces the considerable costs to the health system generated by non-adherence (94).

473 During the WelTel Kenya 1 SMS trial, participants who received SMS support had 474 significantly improved ART adherence and rates of viral suppression compared with control 
Version 3.1, Jan 2021

475 participants (27). In rural Uganda, text messaging was effective with regard to increasing ART

476 adherence among adults, and this study aims to explore this effect among adolescents with poor

477 virological suppression (48).

478 Medication adherence is the "Plus" in the 90-90-90-Plus global challenge, emphasizing the 479 importance of ART adherence in achieving viral suppression. Many gaps remain in meeting these 480 goals, adherence self-efficacy, depression, stressful life events, and perceived stigma were 481 significant predictors of medication adherence (95). Mobile phone applications that support 482 treatment adherence address forgetfulness, knowledge gap; an important barrier to treatment 483 adherence, and this needs to be explored among the youths in Uganda.

\section{4 mHealth and retention in care}

485 Predictors of poor retention in HIV care include younger age, male sex, racial or ethnic 486 minority status, low socioeconomic status, no usual source of health care, less advanced HIV 487 disease, fewer non-HIV-related comorbidities, and greater unmet psychosocial needs (96). Thus, 488 interventions to improve retention in care should incorporate informational, motivational, and 489 behavioral skill components (97).

490 Retaining individuals who have initiated ART in care is challenging. A review by Fox and 491 Rosen 2010, estimated that $20 \%$ of patients in resource-limited settings are lost to follow-up within 49212 months of initiating therapy (98). Prior the era of "test and treat", the ART naïve "ART493 ineligible" patients' clinic retention rate was estimate at 63\% at month 12 (99). There are many 494 challenges for continuity of care, financial constraints, travel difficulties and stigma are factors 495 that can prevent PLWH who start treatment from continuing in care (100).

496 Many people newly diagnosed with HIV are lost to follow-up shortly before or after timely 497 initiation of antiretroviral therapy. One study in Kenya documented that people living with HIV 
Version 3.1, Jan 2021

felt that increased communication via the text messaging intervention had the potential to enable early identification of problems, leading to timely problem solving that could improve retention and engagement in care during the first year after diagnosis. Engaging PLWH at all points along the cascade of care is essential for transforming the management of the disease from a fatal condition to a chronic one $(101,102)$.

In a recently published community-based individual randomized trial in Uganda, no statistically significant differences in retention were observed in the first 6 months between those randomized to either mobile-phone or physical contact tracing reminders. Retention was higher among those who received physical contact reminders at month 12 (91.5\% versus $82.1 \%$; $\mathrm{p}=\mathrm{X}$ ) (103). Although physical contact tracing reminders had higher retention at 12 and 18 months, mobile technology has several advantages which may be cost saving. The challenges of physical contact included structural barriers, time consuming, health care worker exhaustion and physical infrastructure challenges.

Various studies in Uganda have shown that patient-related factors resulting in loss to followup include transportation costs, distance to health centers, inconsistent income and lack of social support. Financing of transport improved retention, but this option is not cost effective nor sustainable in resource poor countries (104-106).

Mobile health interventions may improve engagement in care. Mobile apps provide a convenient vehicle for reaching a large audience and offer the potential to create personalized and interactive interventions that can be used anonymously and discretely. In a study evaluating how youth use technology and mobile apps and how their use of technology could support their health and influence their engagement in care, participants suggested that a mobile health application should have the ability to connect to a community of other youth living with HIV, readily access 
521 healthcare providers, track personal data and information (such as laboratory data), and obtain 522 health news and education (107).

\section{$523 \quad 2.5$ mHealth technologies costs}

524 Information systems, such as electronic health records (EHRs) and mobile phones and 525 handheld computers (also called mHealth), can be of enormous value in providing health care in 526 multiple settings. A review done in 2011 showed that with the exception of personal digital 527 assistant (PDA)-based data collection, there are still few scientifically rigorous data on the 528 effectiveness and cost-effectiveness of e-health systems in developing countries (108). The World 529 Health Organization (WHO) published a manual on implementing EHRs for developing countries.

530 The manual states that a thorough cost-benefit analysis should be conducted to compare the options 531 against each other and the costs of any proposed system against the perceived benefits, so as to 532 determine the value of the system to institution/ government (109).

533 A systematic review of peer-reviewed mHealth literature in Africa published between 2003 534 and 2013 identified 44 studies on mHealth and found that mHealth interventions were associated 535 with positive health-related outcomes. Their success was based on the accessibility, acceptance 536 and low-cost of the technology, effective adaptation to local contexts, strong stakeholder 537 collaboration, and government involvement. In this review, it was clearly noted that threats such 538 as dependency on funding, unclear healthcare system responsibilities, unreliable infrastructure and 539 lack of evidence on cost-effectiveness challenge mHealth implementation (108). 


\section{$541 \quad 2.6$ Hypotheses and Research Questions}

542

543

544

545

546

547

548

549

550

551

552

553

554

555

556

557

558

1. What is the acceptability of mHealth among YLWH at three (03) of Kiryandongo District HIV Centres, and what are the barriers, facilitators/enablers of ART and clinic visit adherence?

Hypothesis: YLWH will have a high acceptability for mHealth with $70 \%$ accepting to use mHealth.

2. What is the effect of the mHealth-CFLU tool on ART adherence among YLWH?

Hypothesis: More than $79 \%$ of YLWH using the mHealth-CFLU tool will be adherent to ART (using viral load suppression as a proxy measure for ART adherence we anticipate an increment of $15 \%)$.

3. What is the cost of the mHealth CFLU intervention compared to standard of care over a12 month period?

Hypothesis: mHealth will be cost effective by increasing proportion in care and proportion with viral suppression compared to standard of care 


\section{3.1 Study Design: Mixed Methods Sequential study}

561 The study design is a mixed method sequential, where the qualitative study will be done first,

562 followed by the randomized controlled trial and thereafter the cost evaluation study.

\section{$563 \quad 3.2$ Study setting}

564 The study will be based at three HIV care and treatment centres in Kiryandongo District (figure $5654)$.

566 Kiryandongo District has 19 government health units (one hospital, 5 Health Centre IIIs and 13 567 Health Centre II) which serve a population over 317,500 people.The study sites will include 568 Kiryandongo Hospital ART clinic, Panyandoli Health Centre III and Nyakadote Health Centre 569 (HC)II. Kiryandongo is located $225 \mathrm{~km}$ on the Kampala -Gulu highway, with a viral load 570 suppression prevalence of 55.3\%(UPHIA, 2017). Kiryandongo hospital has been chosen as the

571 study site, because it is an IDI implementing site with >1000 YLWH in care and a viral suppression 572 rate of $79 \%$ below the UNAIDS target of $90 \%$ (Table 1). In 2018, a total of 637 youths were 573 enrolled in care and retention in care was 79\%. The current total of YLWH at the site is 1,322. The 574 two government centres Panyadoli and Nyakadoti are 44 mins /19 mins drive from Kiryandongo 575 hospital which $30 \mathrm{KM}$ and $16 \mathrm{KM}$ from the Hospital. The HCIII serves a population of over 57680,000 people and are included as they serve a big population of youth living with HIV in the 577 district. 
579 The study population will comprise of YLWH aged 15 -24 years who are ART naïve or on ART $580 \quad$ since Jun 2019.

581 Target population:

582 Youth living with HIV registered to receive ART at any of the 3 ART clinic in Kiryandongo

583 district; Kiryandongo Hospital, Panyandoli HCIII and Nyakadoti HCII

584 Accessible population:

585 Youths consented/assented for CFLU, registered for care at Kiryandongo hospital, Panyadoli 586 Health Centre III and Nyakadoti Health Centre II.

$587 \quad 3.3$ Materials and Methods

\section{Study Objectives:}

589 1a: To assess barriers, enablers of adherence among youth living with HIV in Kiryandongo at 590 baseline and study end.

591 1b: To assess acceptability of mHealth among youth living with HIV in Kiryandongo at baseline 592 and study end.

593 Study design: Qualitative study with narrative approach

594 Study purpose: To identify the key behavioral constructs that enable or hinder ART adherence 595 among the youths, and to assess acceptability of mHealth interventions for adherence support.

596 Study population: YLWH 15-24 years attending Kiryandongo Hospital, Panyadoli Health Centre 597 III or Nyakadote Health Centre II HIV/ART clinic, ART naïve or on ART since Jun 2019. 
598 Sampling: Participants will be purposively selected, each group will include 6-10 YWLH, and 599 there will be a total of 3-5 groups ((110). The participant groups will comprise of those who are 600 naïve or initiating and on ART since Jun 2019. YWLH will be stratified by age group ( $\leq 18 y$ s and $601>18 \mathrm{yrs})$ and gender.

602 Study Procedure:

603 Selection criteria:

604 Inclusion: Youth living with HIV, with a documented HIV test result, aged 15-24 yrs, ART naïve 605 or on ART since Jun 2019.

606 Exclusion Criteria: Youth unable to express themselves verbally, those unable to attend 2 607 sessions of the discussion

608 Selection of participants: We shall liaise with the clinic counsellor to refer YLWH aged 15-24 609 years with a documented HIV test result, patients who are ART naïve or initiating ART and have 610 gone through routine counselling and testing. Stratification by age group and gender will be 611 considered in selection of study participants. Eligible youth will be identified by the clinic 612 counsellor and will be referred to study team which will be located at the HIV clinic. We shall sit 613 one on one with the potential participant, give information about the purpose of the study, and 614 request them to join the focus group discussion on the scheduled date if they agree to take part. 615 This procedure will be repeated until the sample size of a particular group is reached.

616 Sample Size: Focus group discussions will be held until data saturation is achieved.

\section{Data Collection:}


Version 3.1, Jan 2021

618 Focus group discussions: On the day of the discussion, study information will be given to the

619 participant including the purpose of the study, risks and benefits. We will address any questions 620 they may have and obtain consent. An experienced person (Social Sciences group at the Academy)

621 in conducting FGDs will facilitate the discussion assisted by two note takers (the PI and a research 622 assistant). Ground rules will be set and choice of languages to use will be Runyoro, Kiswahili and 623 English which are the dominant languages at the site. FGDs have been preferred and not in-depth 624 interviews, because this study is generating information on collective views which will be used 625 for later, and we want to generate a rich understanding of the youths experiences and beliefs 626 (111).

627 At baseline, we will administer an interview guide consisting of structured questions on the CFLU 628 mHealth tool and ART adherence, as well as open-ended qualitative questions with probes, to 629 allow exploration of YLWH views and opinions regarding these themes. The key issues to be 630 explored are whether the youth would accept or refuse use of an mHealth tool, what they would/ 631 wouldn't like the system to offer in terms of health education, frequency and timing of pill 632 reminders and why. Regarding ART adherence, ART naïve participants will be asked what factors 633 will likely facilitate / hinder their adherence to clinic attendance and ART. ART exposed 634 participants will be asked what has facilitated or hindered their clinic attendance and ART 635 adherence. The FGD will last for about 40 to 45 minutes. It will be audio recorded and 636 refreshments (light snack and a soft drink) will be served. Themes to be assessed at baseline 637 include acceptability of mHealth, assessing knowledge, usage, specifics needed, frequency timing.

638 At the end of the study, the groups will be subdivided according to the themes of acceptability and 639 adherence. For acceptability, further segmentation will be based on those who have utilized the 640 mHealth tool above or below $75 \%$ of times. For ART adherence, we shall subgroup according to 
641 viral suppression (VL <1000 or >1000 copies/mL), gender and age-group below or above 18 years.

642 The FGD guide (appendix IV) will explore the different barriers and enablers for adherence, and 643 the responses to mHealth intervention. The discussion will be captured on an audio recorder and 644 handwritten notes.

645 Data collection for the FGD at study entry, before randomization: Participants' grouping 646 based on ART duration, age and gender at baseline (Table1).

\section{Table 1. Themes and focus groups at base line}

\begin{tabular}{|c|c|c|c|c|}
\hline \multirow{3}{*}{ Themes } & \multicolumn{2}{|c|}{$\begin{array}{l}\text { ART experienced ( } \\
\text { ART 3-months Plus) }\end{array}$} & \multicolumn{2}{|c|}{$\begin{array}{l}\text { Less experienced }(<= \\
\text { 3months })\end{array}$} \\
\hline & \multicolumn{2}{|l|}{ Male } & \multicolumn{2}{|l|}{ Female } \\
\hline & $\leq 18 \mathrm{yrs}$ & $>18 y r s$ & $\leq 18 \mathrm{yrs}$ & $>18 y r s$ \\
\hline
\end{tabular}

\section{Acceptability:}

Why accept or refuse mHealth

How can the system be used for adherence support (probe on time, frequency)?

What they would like the system to do for them( Probe on pill reminders, Health tips/advice, symptom reporting and management and use of secret pin)

\section{Adherence checks:}

Enablers/barriers:

(what has facilitated/hindered for those who are ART experienced)

(what will likely facilitate- for those who are naïve or less experienced 
649 Data collection for the FGD at study end; groups will be divided according to how well they

650 interacted with mHealth tool $(>50 \% \leq 50 \%)$, age group $(>18 \mathrm{yrs} \leq 18 \mathrm{yrs})$ and sex (male or

651 female) (Table 2).

652 Table 2: Themes and groups for FGD at study endline

\begin{tabular}{|c|c|c|c|c|}
\hline \multirow{4}{*}{ Themes } & \multicolumn{4}{|c|}{ Groups } \\
\hline & \multicolumn{2}{|c|}{$\begin{array}{l}\leq 50 \% \\
\text { interaction/engagement } \\
\text { with tool }\end{array}$} & \multicolumn{2}{|c|}{$\begin{array}{l}\text { (>50\% interaction/engagement with } \\
\text { tool) }\end{array}$} \\
\hline & \multicolumn{2}{|l|}{ Male } & \multicolumn{2}{|l|}{ Female } \\
\hline & $\leq 18 \mathrm{yrs}$ & $>18 \mathrm{yrs}$ & $\leq 18 \mathrm{yrs}$ & $>18 y r s$ \\
\hline \multicolumn{5}{|l|}{ Acceptability: } \\
\hline \multicolumn{5}{|l|}{ Comfort/ease using the tool } \\
\hline \multicolumn{5}{|c|}{ Following advice from Health tips } \\
\hline \multicolumn{5}{|l|}{ Likes/dislikes for tool } \\
\hline \multicolumn{5}{|c|}{ Improvements \& recommendations } \\
\hline \multicolumn{5}{|l|}{ Adherence: } \\
\hline \multicolumn{5}{|l|}{ Enablers (what has facilitated) } \\
\hline Barriers( What has hindered) & & & & \\
\hline
\end{tabular}


655 The FGD audio-recordings will be translated and transcribed into English by a group of two

656 qualitative research assistants from the Academy at Infectious Diseases Institute. Transcripts will

657 be read, to identify themes and subthemes. Themes will be discussed, coded and the coded book 658 with the transcripts will be imported to Nvivo for coding and content analysis. The analysis will 659 be done by the qualitative social sciences Lead from the Academy at IDI. Audio files will be 660 electronically stored in password protected files which will only be accessible to the study team.

661 The analysis will be guided by the themes already contained in the interview guide, which will 662 further be refined following multiple readings of interview scripts to better understand the data, 663 identify sub-themes and to group the data according to themes for analysis and interpretation. 664 Quotations reflecting the youths' views, opinions and experiences will be identified and will be 665 used in the presentation of study findings.

Study Objective 2:

667 To assess effect of mHealth tool (CFLU) on ART adherence and knowledge of COVID-19 668 among YLWH naïve, initiating ART or on ART since Jun 2019.

669 Study design: Randomized control trial with two parallel arms (Standard of Care or CFLU with 670 Standard of Care)

671 Study purpose: To assess effect of CFLU on ART adherence among youth measured by 672 interactive voice response to daily adherence calls mapped in the database and proportion with 673 viral suppression of copies below 1000 copies/mL.

674 The intervention (CFLU) uses IVR calls or text messages delivered via MOTECH ${ }^{\mathrm{TM}}$ based 675 Connect for Life technology ${ }^{\mathrm{TM}}$. The calls are delivered in 4 languages (Luganda, Luo English and 
676 Runyakitara) and the participant has to make a choice of the preferred language during registration

677 to the system. The system offers adherence pill reminders, health message tips, visit appointment 678 reminders and receipt of self-reported symptoms.

679 A poor understanding of the disease among youths can result in delayed identification and 680 treatment leading to rapid spread of infections among the community.

681 Study setting: Kiryandongo District, three sites offering HIV-ART. Kiryandongo hospital, 682 Panyandoli Health Centre III and Nyakadote Health Centre II have been chosen as the study sites, 683 the hospital has 1,322 YLWH and a viral suppression rate of 79\% (Table 1). In 2018, a total of 684637 YLWH were enrolled in care. By Jun 2019 and additional 218 YLWH had been enrolled in 685 care. The current total at the site for age between $15-24$ is 1,322 . Overall retention was $79 \%$.We 686 have included the lower level health facilities since ART delivery is done majorly in these 687 grassroot levels.

688 Study Population: Inclusion: YLWH (15-24 years), ART naïve or initiating ART since Jun 6892019.

690 Exclusion: Hearing loss, severe illness likely to shorten life span, boarding school YLWH, 691 Inability to use a basic mobile phone.

692 Sample size calculation:

693 Size: X-Sectional, Cohort, \& Randomized Clinical Trials (Kelsey et al., Methods in 694 Observational Epidemiology 2nd Edition, Table 12-15; Fleiss, Statistical Methods for Rates and 695 Proportions, formulas $3.18 \& 3.19)$

696 The sample size formula for the method described in Kelsey et. al. is: 


\section{$n_{1}=\frac{\left(Z_{w_{2}}+Z_{1 \beta}\right)^{2} \bar{p} \bar{q}(r+1)}{r\left(p_{1}-p_{2}\right)^{2}}$}

697

698 and

$699 \quad \mathbf{n}_{2}=\mathbf{r n}_{1}$

700 where

$701 \mathbf{n}_{1}=$ number of cases in the intervention

$702 \mathbf{n}_{2}=$ number of controls

$703 \quad Z_{\mathbf{n} / 2}=$ standard normal deviate for two-tailed test based on alpha level (relates to the

704 confidence interval level)

$\boldsymbol{Z}_{\boldsymbol{p}}=$ standard normal deviate for one-tailed test based on beta level (relates to the power

706 level)

$707 \quad \mathbf{r}=$ ratio of controls to cases

$708 \mathrm{p} 1=$ proportion of cases with exposure and $\mathrm{q} 1=1-\mathrm{p} 1$

$709 \mathrm{p} 2=$ proportion of controls with exposure and $\mathrm{q} 2=1-\mathrm{p} 2$

$$
\overline{\mathbf{p}}=\frac{\mathbf{p}_{1}+\mathbf{T}_{2}}{\mathbf{r}+\mathbf{1}} \text { and } \overline{\mathbf{q}}=\mathbf{1}-\overline{\mathbf{p}}
$$

711 The sample size formula without the correction factor by Fleiss is: 


$$
n_{\mathbf{k c}}=\frac{n_{1}}{4}\left[1+\sqrt{1+\frac{2(r+1)}{n_{1}\left|p_{2}-p_{1}\right|}}\right]
$$

713 When the input is provided as an odds ratio (OR) rather than the proportion of cases exposed, the

714 proportion of cases exposed is calculated as:

$$
P_{1}=\frac{F_{2} O R}{1+P_{2}(O R-1)}
$$

\section{Sample Size: X-Sectional, Cohort, \& Randomized Clinical Trials (112) \\ (https://www.openepi.com/SampleSize/SSCohort.htm)}

Two-sided significance level (1- $\alpha)$ : $\quad 95$

Power (1- $\beta, \%$ chance of detecting): $\quad 80$

Ratio of sample size, Unexposed/Exposed: 1

Percent of Unexposed with Outcome: $\quad 79$

Percent of Exposed with Outcome: $\quad$ 94(83)

Odds Ratio:

Prevalence Ratio:

1.2

Prevalence difference: 


\begin{tabular}{llll}
\hline Sample size- Exposed(intervention): & 81 & 80 & 93 \\
Sample Size-Nonexposed (Control): & 81 & 80 & 93
\end{tabular}

Total Sample Size: $\quad 162 \quad 160 \quad \mathbf{1 8 6}$

$716 \mathrm{CC}=$ continuity correction. Results are rounded up to the nearest integer.

717 Factoring 10\% loss to follow-up, the total sample size will be 206(103 for each arm)

718 Data collection procedures: Figure 5, illustrates the study schema, from screening to enrollment 719 and data collection.

721 observed that a good number of participants (50 of 256) at the urban site were not enrolled due to 722 absence of a basic mobile phone, or had malfunctioning phone keyboards (for Poster Exhibition 723 at the 20th International Conference on AIDS and STIs in Africa (ICASA 2019). Therefore, we

724 shall provide a basic mobile phone to everyone in the study (SoC and intervention arm). To avoid 725 stigma associated with a particular phone, we shall get 4 or more types of basic phones. Issues 726 regarding simcard registration will be dealt with by the individuals.

727 Four study questionnaires will be administered at baseline and 6-monthly until the 12-month exit 728 visit.

729 The questionnaires will capture:

730 - Sociodemographic and medical history

731 - Stigma scores

732 - Knowledge assessment

733 - Sexual behavior assessment 
735 The data will be entered using REDCap. Blood for viral load will be collected at baseline and at 736 the 6 and 12-month visit.

\section{Intervention: mHealth Call For Life Uganda tool}

The tool capitalises on a basic mobile phone's core utilities of voice and short messaging

739 services. The system design, development, testing and evaluation was done by Janssen and the

740 Grameen Foundation (http://motechsuite.org/index.php/implementations). In 2015, Janssen

741 Global Public Health Research and Development, in close collaboration with the Infectious

742 Diseases Institute Kampala (IDI), developed Call for Life Uganda (CFLU) tailored to the needs of

743 PLHIV in Uganda. The CFLU system follows the Health Insurance Portability and Accountability

744 Act (HIPAA) Privacy and Security rules. While the Privacy rules deals with electronic Protected

745 Health Information, the security rule covers administrative, physical and technical safeguards to

746 ensure confidentiality, integrity and security of electronic protected health information. Data

747 transaction between patient and system is encrypted when it comes to human subjects. The system

748 is password protected, and interaction of system and patient is personalised with a secret code. The

749 system interacts with the patient through a basic mobile phone via a keypad and with the health

750 worker through a web-based interface. The system has options to either use interactive voice

751 response or short message service and the user has to make a choice, get a secret pin code which

752 ensures privacy to enduser.

\section{Screening-Enrolment procedures:}

All potential participants will be written on the screening log, and a screening form

755 completed, reasons for screen failure will be documented on the screening log. Following 
Version 3.1, Jan 2021

756 successful screening, the participant will be referred to the Research Assistant (RA) to receive a

757 thorough explanation of the study and informed consent is obtained. The RA then administers the 758 stigma questionnaire along with the sociodemographic and medical information, , HIV knowledge 759 and sexual behaviour questionnaires. A client locater form will be completed which will help in 760 physical contact tracing, and the participant will be registered on the enrolment log.

Randomization will follow (see details of randomization procedure-standard operating

762 procedure) thereafter and the result in the sealed envelope will dictate whether the patient will be 763 registered onto the Call for Life System for the intervention or continue with standard of care as 764 per MoH HIV Management guidelines.

765 Registration onto the CFLU system: Refer to Standard operating procedures on registration.

766 However, all patients will be registered onto the electronic and hard copy of the enrolment log and 767 a next appointment date is given to them. Finally, the lab request forms are filled and the patient 768 sent for a blood draw. The patient returns from the lab, receives their transport refund of UGX 76920,000 , with a basic mobile phone (only at baseline visit, to all participants) and is allowed to go 770 home.

771 No intervention: Standard of care arm (SOC)

772 The Ministry of Health developed a minimum healthcare services package for PLHIV to 773 standardize the programming, implementation and delivery of integrated HIV services in Uganda

774 The standard of care arm is based on the Apr 2018, consolidated guidelines for prevention and 775 treatment of HIV in Uganda (113), and will also follow the healthcare services package for PLHIV 776 (114).

777 The SOC arm will follow the services below: 
Version 3.1, Jan 2021

778 - Care and support for people living with HIV, and the first line ART regimen is

$779 \quad \mathrm{TDF} / 3 \mathrm{TC} / \mathrm{DTG}$

780 - First-Line Regimen for Adults and Adolescents aged above 10 Initiating ART:

781 Preferred First-Line Regimen: TDF+3TC+DTG All HIV-infected adults and adolescents aged 10

782 years and above should be initiated on tenofovir, lamivudine and dolutegravir (TDF+3TC+DTG)

783 as a once-daily fixed dose combination. When to use TDF+3TC+EFV: Adults and adolescents

784 aged 10 years and above should only be initiated on TDF+3TC+EFV if their weight does not

785 allow for use of the currently available DTG formulations (containing 50mg). Note: DTG 50mg

786 should only be given to adults and adolescents weighing 40kg and above. When the $10 \mathrm{mg}$ and

787 25mg formulations become available, DTG will be administered to children and adolescents

788 weighing less than $40 \mathrm{~kg}$.

789 - When to use $\mathrm{ABC}+3 \mathrm{TC}+\mathrm{DTG}$ :

790 Adults and adolescents aged 10 years and above should only be initiated on ABC+3TC+DTG if

791 TDF is contraindicated, including the following conditions:

792 1. Kidney disease and estimated glomerular filtration rate (GFR) below $60 \mathrm{ml} / \mathrm{min}$

793 2. Adolescents below $35 \mathrm{~kg}$ of weight.

794• Adherence Preparation, Monitoring, And Support:

795 The study team will use the 5 As principles for chronic care as a guide to offer pre-ART

796 adherence counselling and psychosocial support. These are Assess, Advise, Agree, Assist and

797 Arrange. The youths will be followed-up every 6 months as per MoH guidelines.

798 The standard Adult Care and Treatment Package includes the following services:(114)

799 1. HIV counselling and testing 
801 3. Nutritional Counseling and support

802 4. Opportunistic infection screening and management

803 5. Screening and management of non-communicable diseases

804 6. Diagnostics and laboratory monitoring

805 7. Sexual and reproductive health

806 8. Counseling and Psychosocial support

807 9. ARV preparation, initiation and monitoring

808 10. Adherence and retention into care

809 11. Palliative care

810 12. Mental health

\section{The outcome variables include:}

812 - ART adherence scores at 6 and 12 months, as assessed by HIV Care Card for those on SoC

813 arm and Self- reported adherence to ART as depicted on the mHealth dash board.

814 - Viral load suppression at 6 and 12 months, as test results from blood collected at months 6

$815 \quad$ and 12 with time to viral suppression.

816 Retention rates at 6 and 12 months with time to loss to follow-up

817 - Stigma scores at study start and end

818 - HIV Knowledge and sexual behaviour assessment at study start and end 
820 Data collected on paper CRF will be entered using Research Electronic Data Capture software

821 (REDCap) which is a safe, secure, HIPAA-compliant, web-based application and facilitates offline

822 and online data entry. Data will be exported to STATA 14.0, for analysis. Data will then be backed

823 up and archived in both soft and hard copy to avoid losses. The data collected will be stored

824 securely under lock and key to maintain patient confidentiality.

825 Data regarding call details in the system will be extracted from Call For Life tool database, 826 exported into Stata for analysis.

827 Visit dates will be extracted from database and proportions lost to follow-up drawn and time to

828 loss to follow-up estimated. Viral load results will be extracted and time to viral suppression with 829 survival analysis curves drawn for both arms.

830 Data from the Ministry of Health HIV card regarding the ART regimen and start dates will be 831 transcribed to the data collection tool and later entered on the REDCap system.

\section{General data analysis}

833 Data will be analyzed at three levels, first at the univariate level, then at the bivariate level and 834 lastly at the multivariate level. A consort diagram will be drawn to show the screening, enrolment, 835 randomization, follow-up and analysis flow of the study.

\section{Univariate Analysis}

837 Descriptive analysis will be done for the factors that influence adherence, virological suppression 838 , stigma and retention in care. In addition, the distribution of the independent variables will be 839 done with categorical data summarized using frequencies and percentages. Numerical data will be 
840 summarized using means and standard deviations for normally distributed data, and median and

841 inter-quartile range for data that is not normally distributed.

\section{$842 \quad$ Bivariate Analysis}

843 The relationship between independent variables (age, gender, ART duration, marital status, 844 education status, occupation and religion) and adherence and virological suppression will be 845 analyzed using bivariate analysis made using cross tabular analysis and associations investigated 846 using the Pearson Chi-square test.

847 The Chi-square test is based on the computational formulae below:

$$
\chi^{2}=\sum_{\mathrm{i}=1 \mathrm{j}=1}^{\mathrm{r}} \sum_{\mathrm{ij}}^{\mathrm{c}} \frac{\left(\mathrm{O}_{\mathrm{ij}}-\mathrm{E}_{\mathrm{j}}\right)^{2}}{\mathrm{E}_{\mathrm{j}}}
$$

$849 \quad$ Where

$850 \mathrm{r}$ is the number of categories of the $\mathrm{i}^{\text {th }}$ explanatory variable

$851 \quad \mathrm{c}$ is the number of categories of the dependent variable

O ${ }_{\mathrm{ij}}$ is the observed number of observations in the $\mathrm{i}^{\text {th }}$ explanatory and jth dependent variable and $\mathrm{j}^{\text {th }}$ dependent variable 
856 Further, odds ratios shall be used to analyze the relationship between the dependent variable and

857 the independent variables. Variables found to be significant at $p$ value of $p \leq 0.05$ will considered 858 for the multivariable analysis.

\section{$859 \quad$ Multivariate Analysis}

860 Multivariable logistic regression will be used to determine the factors that influence adherence or

861 virological suppression and retention in care. Variables found to be statistically significant at $\mathrm{p} \leq$

8620.05 in bivariate analysis and those considered to have a scientific explanation will be entered

863 into the multivariable model.

864 The multivariate logistic regression adopted is based on the formulae below:

865

$\ln \left[\frac{\mathrm{p}_{\mathrm{i}}}{1-\mathrm{p}_{\mathrm{i}}}\right]=\beta_{0}+\beta_{1} \mathrm{X}_{1}+\ldots+\beta_{k} \mathrm{X}_{\mathrm{k}}$

Where;

$867 \mathrm{X}_{\mathrm{i}}=$ independent variables

$868 \mathrm{p}_{\mathrm{i}}=$ Probability of being adherent and viralogically suppression

$869 \quad 1-p_{i}=$ Probability of not non-adherence or not suppressed virologically

$870 \beta_{0}=$ constant $; \beta i=$ coefficient of the determinant

871 The study findings will be reported as odds ratios, confidence interval and independent variables

872 found to have $p$ value $\leq 0.05$ at $95 \%$ confidence interval will be considered to be statistically

873 significant in the final model. 
Version 3.1, Jan 2021

874 After the final model, significant factors for ART adherence/ viral suppression will be computed.

\section{Data Monitoring Committee:}

876 The DMC will consist independent members with expertise in relevant clinical specialties, and 877 statistical data management the PhD supervisors will be among the committee. Their responsibility 878 is to ensure that the safety of study subjects is protected while the scientific goals of the study are 879 being met, and generate data queries to study team. The previous CFL-RCT at IDI, had no major 880 safety issues and neither were there any gender-based violence, so the DMC will suffice and will 881 be involved in:

- Review the research protocol and plan for data safety and monitoring.

- Generate data queries at a regular interval from the REDCap website

- Evaluate the progress of the trial, including periodic assessments of data quality and timeliness, participant recruitment, accrual and retention, participant risk versus benefit, performance of the trial site, and other factors that can affect study outcome.

- Make recommendations to the PI about continuation, termination, or other modifications of the trial based on the observed beneficial or adverse effects of the intervention under study.

- If appropriate, conduct interim analysis of efficacy in accordance

- Recommend solutions to address problems with study conduct, enrolment, and sample size and/or data collection.

\section{Study visits (scheduled and unscheduled visit codes)}


894 The total duration of the study is 18 months; follow up period is up to 12 months. The participant 895 has a baseline visit (00.0) and is followed up at month 6 (168 days \pm 14 days) visit code 01.0 , last 896 visit is at month 12 (336 days \pm 14 days) visit code 02.0 .

897 The participant might return at the clinic due to an illness, or repeat blood draw, this interim visit 898 codes will be numbered sequentially from the previous scheduled visit. $(00.01,00.02$ if its next to 899 the baseline, if next to second visit, the code will be $01.01,01.02$ etc., if its next to last visit it will 900 be coded $02.1,02.2$, etc.).

901 The study visit window is 2 weeks (14 days), beyond this period a visit code denoting an interim 902 visit is given; e.g. Visit code 01.1 first contact with patient past visit 01.0 and 01.2 denotes second 903 contact with patient past visit 01.0 .

904 During enrolment, it is important to obtain the names and contact information for several 905 individuals closely related to the participant (e.g., next of kin, friends, etc.). Such individuals can 906 be contacted in the event that a participant does not return for follow-up visits. Physical contact 907 tracing will happen if the patient takes 2 weeks past the scheduled visit.

908 Table 3: Study schedule of procedures: 
Version 3.1, Jan 2021

\begin{tabular}{|c|c|c|c|}
\hline Study schedule of procedures & $\begin{array}{l}\text { Baseline Month } \\
00+2 \text { weeks }\end{array}$ & $\begin{array}{l}\text { Month } 6 \pm 2 \\
\text { weeks }\end{array}$ & $\begin{array}{l}\text { Month } 12 \pm 2 \\
\text { weeks }\end{array}$ \\
\hline Eligibility Screening & $\sqrt{ }$ & & \\
\hline Informed Consent & $\sqrt{ }$ & & \\
\hline $\begin{array}{l}\text { Barriers, enablers of adherence and } \\
\text { acceptability (Qualitative data } \\
\text { collection at baseline) }\end{array}$ & $\sqrt{ }$ & & \\
\hline $\begin{array}{l}\text { Randomization : Intervention (Call for } \\
\text { life mHealth IVR) with Standard of care } \\
\text { Versus Standard of care alone }\end{array}$ & & & \\
\hline COVID-19 Knowledge Assessment & $\sqrt{ }$ & & \\
\hline Stigma Scores & $\sqrt{ }$ & & $\sqrt{ }$ \\
\hline $\begin{array}{l}\text { - Demographic and socio- } \\
\text { economic assessment, } \\
\text { - Knowledge questionnaire, } \\
\text { - Sexual behavior questionnaire }\end{array}$ & $\sqrt{ }$ & $\sqrt{ }$ & $\sqrt{ }$ \\
\hline Randomization & $\sqrt{ }$ & & \\
\hline Laboratory & & & \\
\hline Viral load \pm CD4* & $\sqrt{ } *$ & $\sqrt{ }$ & $\sqrt{ }$ \\
\hline Resistance testing(If funds allow) & & & $\sqrt{* *}$ \\
\hline Qualitative assessment-study end-line & & & $\sqrt{ }$ \\
\hline
\end{tabular}

*Those initiating or ART naïve at study entry ** Depending on availability of funds

911 CD4: cluster differentiation four 
913 Missed visit:

914 When the appointment is not honoured and the 14-day window passes, this is a missed visit. If a 915 participant misses a visit (Visit window inclusive) a missed visit form is completed documenting 916 the reason for missing (if known) and the attempts made to contact the participant, through mobile

917 phone or physical tracing. Several attempts to contact the participants must be made to ensure good 918 retention. The attempts may include: Checking the General clinic schedule to sync the appointment 919 visit, Calling the participant; Calling a relative who was disclosed to, or Visiting the participant's 920 home: To minimise missed visits, through the web-interface on CFLU system, any dropped calls 921 monitored on the dashboard, will trigger active follow-up and tracing of the participants.

923 Loss to follow-up:

924 If a participant misses 2 consecutive visits and unable to ascertain the vital status, this patient will 925 be declared lost to follow-up, alternatively, if 3 months consecutively elapse, without 926 acknowledging the patient's status, the patient will be labelled lost to follow-up. If a participant's 927 where about is not known for 3 months and the contact tracing is uneventful after 3 documented 928 attempts, this participant is deemed lost to follow-up and a withdraw form is completed. In case 929 the participant re-surfaces to the clinic when the study is still active/ongoing, the withdraw form 930 is cancelled out and patient re-joins the study and a study visit is conducted.

\section{Study Objective 3:}

932 To evaluate the cost of mHealth in comparison to Standard of Care

933 Study design: Cost evaluation analysis 
934 Study Purpose: To assess cost of mHealth intervention in comparison to standard of care

935 Primary outcome measure: Costs spent on the intervention participants in comparison to

936 standard of care participants

937 A cost analysis of the intervention from a governmental perspective will be performed. The 938 principle of marginal costing will be used to cost the resources utilised in the alternative strategies.

939 All costs of a historical nature, the sunk costs such as resources invested in buildings, equipment, 940 and vehicles will be ignored. Only costs related to the future, from the onset of the study, will be 941 recorded.

942 Sources of cost information: The following costs will be assessed and classified under direct or 943 indirect cost: Sources of cost data will be; program/ project documents, financial records.

944 Capital costs:

I. System Costs:

- Software monthly hosting (internet, cloud hosting, call bills,)

- Support maintenance

948

- Hard ware

○ Computers

○ Internet modem

\section{Recurrent costs:}


955 Induced costs:

I. Transport costs

II. Number of sick visits attended outside the clinic costs/fees incurred

III. Airtime fees

\section{Health outcomes:}

960 The number of patients with viral suppression (<1000 copies) will listed per study arm at month 6

961 and 12, and these will be compared across arms.

962 Analysis:

963 Primary measure will be Cost for mHealth Intervention.

\section{$964 \quad 3.5$ Quality control}

965 All data collection tools will be checked for completeness and validated prior to entry on REDCap.

966 Research assistants (research nurse counsellors) will follow the research protocol, have prior 967 protocol training, Human subject protection (HSP), and Good Clinical practice (GCP) course 968 training. After randomization the participant on the intervention (CFL arm) will be registered on 969 a standalone CFL-PhD clinic for this study. The viral load results entered on the CFL tool will be 970 cross-checked and verified by the second research nurse -counsellor, as the second data entrant. 971 All patients consented for the study will get a basic phone to eliminate issues regarding phone 972 technologies such as some buttons not functioning well. The study will be over seen by the DMC 973 based at infectious Diseases Institute, the PhD supervisors who review the student's progress on 
Version 3.1, Jan 2021

974 the quarterly period, and these supervisors are part of doctoral committee. The internal monitor

975 provided by the sponsor is responsible for bi annual monitoring of the study.

976 Data Security: All study data will be double encrypted, maintaining the confidentiality and safety

977 of all data communications. The communication between the call for life browser and the server

978 is encrypted using 128-bit SSL. System servers are hosted by Amazon Web Services (AWS) and

979 are secured by Amazon VPC and AWS WAF firewalls to allow private networks and prevent

980 unauthorized access, while data is protected from virus threats using BitDefender anti-virus

981 technology. The YLWH database will be housed on the IDI server.

982

983 Study limitations:

984 The intervention involves information technology and requirs reliable electricity supply, stable

985 mobile phone network, and internet connectivity. Any disruption will lead to the CFLU system

986 not operating as planned.

987 For the end user lack of experience with use of pin codes, multiple sim lines and failure to register

988 simcard may lead to system failure Because entry of a wrong pin code will lead to blockage from

989 use of the system. Use of multiple sim cards may lead to sim swapping and a call reminder may

990 be received when the registered/authorised sim card number is not available because the other line

991 is in use. Finally, unregistered simcards may be blocked by the Uganda Communications

992 Commission.

993 Another limitation may be parental restrictions in phone use for YLWH enrolled in the study. The

994 study participant's response to daily pill reminders calls assumes that self-reporting is accurate.

995 The Call for life health messages are in 4 main languages, due to lack of funds, the Swahili

996 language is missing and this may affect many potential participants. 
Version 3.1, Jan 2021

997 Discussion: This project will determine acceptability, effectiveness of mHealth, knowledge \& 998 myths on COVID-19 and cost of delivering pill and clinic appointment reminders, and voice messages to a population with suboptimal ART adherence in a resource-limited setting.

Trial status

1002 The protocol current version number and date is 3.1, Jan 2021, the date recruitment began was

$100312^{\text {th }}$ Aug 2020 and recruitment ended $30^{\text {th }}$ Jun 2021. We are still following up the study

1004 participants, and the last follow-up will be $30^{\text {th }}$ May 2022. I was not able to publish the protocol

1005 earlier, this is because the country underwent lockdown due to COVID-19 pandermic, just after

1006 receiving regulatory approval, when lockdown was eased, I put emphasis on recruitment to

1007 catch up with the PhD timelines and the funding period expiring in the next year.

1008 This protocol has been ammended twice, as explained below:

1009 First amendment on version

1010

1011

1012

1013

1014

1015

1016

1017

1018

1019
- Revised the study population and subject selection to include youth who initiated ART

by Jun 2019.Previously the study population was Youth who are ART naive, initiating or on ART for $\leq 3$ months, this was revised to ; youth who are ART naive, initiating or on ART since Jun 2019.

- Added a new questionnaire to assess knowledge of and myths about COVID-19 among youths in rural area

\section{Reason for first amendment/modification on version 2.2, Jan 2020:}

Due to COVID-19 lockdown, disruption of normal service delivery and impact it has had on health seeking behaviour, we realised that the youth coming to Kiryandongo hospital for HIV testing and thus initiating ART markedly declined in the past 3 months. This was likely to 
1020 impact on the recruitment and timelines for this $\mathrm{PhD}$ study, and hence the need to amend the 1021 protocol.

1022

1023

1024

1025

1026

1027

1028

1029

1030

1031

1032

1033

1034

1035

1036

1037

1038

1039

1040

1041

1042
1. To increase on the enrolment rate of this study, the subject selection has been amended.

2. We have also used this opportunity while amending to add a new questionnaire to assess knowledge of and myths about COVID-19 amongst this population.

\section{Second amendment/modification on version 3.0,Jul 2020}

Revised the study sites to include two more low level facilities ( Health Centre level III

and II) near Kiryandongo Hospital.Previously the study site was only at Kiryandongo

Hospital

Reason for second amendment/modification:

Due to COVID-19 lockdown, there was disruption of normal service delivery and most patients requested to get ART transfer out to sites nearer their home. This led to low recruitment and delays to sample size accrual and hence the need to amend the protocol.

1. To increase on the enrolment rate for study, we requested addition of two sites which are Panyandol Health Centre and Nyakadot Health Centre which are within Kiryandongo district. Inclusion of low level health facility involved in HIVcare

\section{List of abbreviations}

AIDS: Acquired Immuno Deficiency Syndrome

cART: Combined Antiretroviral Therapy 
1043 CD4 cell: Cluster Differentiated cells

1044 cDOT: Community based Directly Observed Treatment

1045 CFLU: Call for Life Uganda

1046 CITI: Collaborative Institutional Training Initiative

1047 COVID-19: CORONA VIRUS DISEASE 2019

1048 GCP: Good Clinical Practice

1049 HIT: Health Information Technology

1050 HIV RNA: Human Immunodeficiency Virus Ribonucleic Acid

1051 HSP: Human Subjects Protection

1052 IAC: Intensive Adherence Counselling

1053 IDI: Infectious Diseases Institute

1054 IVR: Interactive Voice Response

1055 MoH: Ministry of Health

1056 PEPFAR: President's Emergency Plan for AIDS Relief

1057 PHS: Physical Health Scores

1058 PLWH: People living with HIV

1059 SMS: Short Messaging Service

1060 UBOS: Uganda Bureau of Statistics

1061 UNAIDS: United Nations Programme on HIV and AIDS

1062 UNCST- Uganda National Council of Science \& Technology

1063 UPHIA: Uganda Population-Based HIV Impact Assessment

1064 VL: Viral load

1065 YLWH: Youth living with HIV 
1067 Permission to carry out the study will be sought from the Department of Medicine, School of 1068 Medicine, College of Health Sciences and ethical approval from the Higher Degrees Ethics and 1069 Research Committee of School of Medicine, Makerere University College of Health Sciences and 1070 research clearance from Uganda National Council for Science Technology. Clearance will also be 1071 sought from Kiryandongo Hospital administration, Health centre in-charges, Kiryandongo District 1072 Health Office and Infectious Diseases Institute -scientific research committee. The PI is Good 1073 Clinical Practice (GCP) compliant with a CITI certificate in GCP and Human Research Subject 1074 Protection (HSP). Informed consent and assent will be obtained and documented from all 1075 participants. Confidentiality will be maintained at all times. All data collected will be secured 1076 under lock and key. The CFLU is password protected and data is encrypted once extracted from 1077 database.

1078 The National IT Authority policy will be followed and adhered to in relation to data protection, 1079 safety and confidentiality for patients.

1080 Consent for publication

1081 Not applicable

1082 Availability of data and materials

1083 The principle investigator will have access to the final trial datasets and can be availed on request, 1084 after signing the agreement from the study sponsor who is Infectious Diseases Institute.

1085 Competing interests

1086 There are no competing interests for all the authors

1087 Funding

1088 ABN received funding for a scholarship from the Academy at Infectious Diseases Institute- The 
1089 scholarship covered university tuition, student stipend and research.

1090 Authors' contributions

$1091 \mathrm{ABN}$ is the Principle Investigator, $\mathrm{PhD}$ student at Makerere University, conceived the study 1092 developed concept, study design, proposal development, protocol writing, ethical and regulatory 1093 approvals.

1094 RPR, DBM, PW, and RJ are PhD supervisors at Makerere University, provide scientific overview, 1095 give guidance on study designs, methodology, edited and reviewed protocol until final approval. 1096 Provide progress assessment of the student.All authors read and approved the final manuscript.

1097 Acknowledgements

1098 I acknowledge MSN, the statitician who helped in generating the randomization list for the trial.

1099 Authors' information

1100 The Author is a PhD student at Makerere University, College of Health Sciences, School of 1101 Medicine, she got a scholarship from the Academy for hhealth Innovations at Infectious Diseases 1102 Institute. 


\section{REFERENCES}

1105

https://www.hiv.gov/hiv-basics/overview/data-and-trends/global-statistics UNAIDS. UNAIDS Data FactSheet. 2018. Avert.Org. Young People HIV and AIDS 2019 [updated 21 Aug 2018; cited 20197 Oct]. UNAIDS. Youth and HIV. 2018. UNAIDS. Global AIDS Update 2019. 2019. AIDSInfo. Young People (15-24yrs) living with HIV-by sex 2019 [cited 2019]. Available from: https://aidsinfo.unaids.org/.

UNAIDS. Ending the AIDS epidemic among children, adolescents and young women 2019 [updated 2019; cited $\quad 2018$ 27 Apr]. Available from: https://www.unaids.org/en/resources/presscentre/featurestories/2018/april/ending-aids-epidemicamong-children-adolescents-young-women.

UNAIDS. 90-90-90 An ambitious treatment target to help end the AIDS epidemic. 2014. UPHIA. UGANDA POPULATION-BASEDHIV IMPACT ASSESSMENT. 2017.

Nabukeera-Barungi N, Elyanu P, Asire B, Katureebe C, Lukabwe I, Namusoke E, et al. Adherence to antiretroviral therapy and retention in care for adolescents living with HIV from 10 districts in Uganda. BMC Infectious Diseases. 2015;15(1):520.

WHO. mHealth: New horizons for health through mobile technologies. 2011.

Musiimenta A, Atukunda EC, Tumuhimbise W, Pisarski EE, Tam M, Wyatt MA, et al. Acceptability and feasibility of real-time antiretroviral therapy adherence interventions in rural Uganda: Mixed-method pilot randomized controlled trial. JMIR Mhealth Uhealth. 2018;6(5):e122. 
1126 Tool for Treatment, Care and Management of Persons Living with HIV. AIDS and behavior. 2015;19 Suppl $11272(02): 81-9$.

1128 Haberer JE, Musiimenta A, Atukunda EC, Musinguzi N, Wyatt MA, Ware NC, et al. Short message 1129 service (SMS) reminders and real-time adherence monitoring improve antiretroviral therapy adherence 1130 in rural Uganda. Aids. 2016;30(8):1295-300.

1131 Campbell AR, Kinvig K, Côté HC, Lester RT, Qiu AQ, Maan EJ, et al. Health Care Provider Utilization 1132 and Cost of an mHealth Intervention in Vulnerable People Living With HIV in Vancouver, Canada: 1133 Prospective Study. JMIR Mhealth Uhealth. 2018;6(7):e152.

1134 Rosalind M. Parkes-Ratanshi, Maria S. Nabaggala, Agnes N. Bwanika, Mohammed Lamorde, 1135 Rachel King, Noela Owarwo, et al. CALL FOR LIFE UGANDA TM: An RCT Using Interactive Voice Response 1136 for PLHIV on ART. Abstract 1037. . Conference on Retroviruses and Opportunistic Infections,; March 4-7; 1137 Seattle2019.

1138 CDC. Report of the NIH Panel to Define Principles of Therapy of HIV Infection and Guidelines for 1139 the Use of Antiretroviral Agents in HIV-Infected Adults and Adolescents. U.S. DEPARTMENT OF HEALTH 1140 AND HUMAN SERVICES

1141 Centers for Disease Control and Prevention (CDC), SERVICES USDOHAH, (CDC) CfDCaP; 1998 Apr 24. 1142 Report No.

1143 Vreeman RC, Nyandiko WM, Liu H, Tu W, Scanlon ML, Slaven JE, et al. Measuring adherence to 1144 antiretroviral therapy in children and adolescents in western Kenya. J Int AIDS Soc. 2014;17(1):19227.

1145 Hawkins A, Evangeli M, Sturgeon K, Le Prevost M, Judd A, Committee AS. Episodic medication 1146 adherence in adolescents and young adults with perinatally acquired HIV: a within-participants approach. 1147 AIDS care. 2016;28(sup1):68-75. 
1148 Gardner EM, Burman WJ, Steiner JF, Anderson PL, Bangsberg DR. Antiretroviral medication

1149 adherence and the development of class-specific antiretroviral resistance. AIDS (London, England).

$1150 \quad$ 2009;23(9):1035.

1151 Oyugi JH, Byakika-Tusiime J, Ragland K, Laeyendecker O, Mugerwa R, Kityo C, et al. Treatment

1152 interruptions predict resistance in HIV-positive individuals purchasing fixed-dose combination

1153 antiretroviral therapy in Kampala, Uganda. Aids. 2007;21(8):965-71.

1154 WHO. Guidelines on HIV self-testing and partner notification; Suppliment to consolidated 1155 guidelines on HIV testing services. . 2016.

Koirala S, Deuba K, Nampaisan O, Marrone G, Ekström AM, group C-S. Facilitators and barriers for

1157 retention in HIV care between testing and treatment in Asia-a study in Bangladesh, Indonesia, Lao, 1158 Nepal, Pakistan, Philippines and Vietnam. PLoS One. 2017;12(5):e0176914.

1159 Hudelson C, Cluver L. Factors associated with adherence to antiretroviral therapy among 1160 adolescents living with HIV/AIDS in low-and middle-income countries: a systematic review. AIDS care. $1161 \quad 2015 ; 27(7): 805-16$

1162 Agwu AL, Bethel J, Hightow-Weidman LB, Sleasman JW, Wilson CM, Rudy B, et al. Substantial 1163 multiclass transmitted drug resistance and drug-relevant polymorphisms among treatment-naïve 1164 behaviorally HIV-infected youth. AIDS patient care and STDs. 2012;26(4):193-6.

1165 Lawrence Mbuagbaw, Lahana Thabane, Pierre Ongolo-Zogo, Richard T Lester, Edward Mills, 1166 Jimmy Volmink, et al. The cameroon mobile phone sms (CAMPS) trial: a protocol for a randomized 1167 controlled trial of mobile phone text messaging versus usual care for improving adherence to highly active 1168 anti-retroviral therapy Trials. 2011;12(5).

1169 Ammassari AM, et al. Timed Short Messaging Service Improves Adherence and Virological 1170 Outcomes in HIV-1-Infected Patients With Suboptimal Adherence to Antiretroviral Therapy. JAIDS. $1171 \quad 2011 ; 58(4):$ e113-e5. 

message service on antiretroviral treatment adherence in Kenya (WelTel Kenya1): a randomised trial. The

1174 Lancet. 2010;376(9755):1838-45.

1175 van der Kop ML OD, Patel A, et al. The effect of weekly short message service communication on 1176 patient retention in care in the first year after HIV diagnosis: study protocol for a randomised controlled 1177 trial (WelTel Retain). BMJ. 2013.

1178 Natalie Leon HSaED. Applying a framework for assessing the health system challenges to scaling 1179 up mHealth in South Africa. BMC Medical Informatics and Decision Making. 2012;12(123).

1180 Garofalo R, Kuhns LM, Hotton A, Johnson A, Muldoon A, Rice D. A Randomized Controlled Trial of 1181 Personalized Text Message Reminders to Promote Medication Adherence Among HIV-Positive 1182 Adolescents and Young Adults. AIDS and behavior. 2016;20(5):1049-59. Byonanebye DM NM, Naggirinya AB, Lamorde M, Oseku E, King R, Owarwo N, Laker E, Orama R,

1184 Castelnuovo B, Kiragga A, Parkes-Ratanshi R. An interactive voice response software to improve the 1185 quality of life of people living with HIV in Uganda: A Randomized Controlled Trial. JMIR mHealth and $1186 \quad$ uHealth. 2021. interactive voice response. Australian and New Zealand Journal of Public Health. 2005;29(3):261-4. Schroder KE, Johnson CJ, Wiebe JS. Interactive Voice Response Technology applied to sexual 1190 behavior self-reports: a comparison of three methods. AIDS and behavior. 2007;11(2):313-23. Perrine MW, Mundt JC, Searles JS, Lester LS. Validation of daily self-reported alcohol consumption 1192 using interactive voice response (IVR) technology. Journal of Studies on Alcohol. 1995;56(5):487-90. Hettema JE, Hosseinbor S, Ingersoll KS. Feasibility and Reliability of Interactive Voice Response 1194 Assessment of HIV Medication Adherence: Research and Clinical Implications. HIV clinical trials. 1195 2012;13(5):271-7. 
1197 improve medication refill compliance. BMC medical informatics and decision making. 2008;8(1):46.

Behavior. Behavioral Aspects of HIV Management. 2009:210- 6.

$$
\text { UPHIA. UGANDA POPULATION-BASED HIV IMPACT ASSESSMENT UPHIA 2016-2017. } 2017 .
$$

UNAIDS. STATE OF THE AIDS EPIDEMIC-UNAIDS DATA 2017. 2017.

1213 HIV Treat m e n t C o n t in u u m Journal of Acquired Immune Deficiency Syndromes. 2017;74:S51214 S14.

1216 in a low socio-economic urban setting in Cape Town, South Africa: University of the Western Cape; 2017. suppression rates following the intensive adherence counseling (IAC) program for children and 
1221 Service Improves Adherence and Virological Outcomes in HIV-1-Infected Patients With Suboptimal

1222 Adherence to Antiretroviral Therapy. JAIDS Journal of Acquired Immune Deficiency Syndromes. $1223 \quad 2011 ; 58(4):$ e113-e5. and Feasibility of Real-Time Antiretroviral Therapy Adherence Interventions in Rural Uganda: Mixed1226 Method Pilot Randomized Controlled Trial. JMIR Mhealth Uhealth. 2018;6(5):e122.

WHO. Consolidated guidelines on the use of antiretroviral drugs for treating and preventing HIV

1228 infection 2013.

1229 50. UBOS. UGANDA BUREAU OF STATISTICS : 2017 Statistical_Abstract: Communication Statistics. 12302017.

Jacob PR, Oates. Cell-Phone in Africa: Communication LifelineAL-April-15-2015. Pew-Research-

1232 Center-Africa. 2015.

Ippoliti NB, L'Engle K. Meet us on the phone: mobile phone programs for adolescent sexual and reproductive health in low-to-middle income countries. Reprod Health. 2017;14(1):11-.

Fisher JD, Fisher WA. The information-motivation-behavioral skills model. Emerging theories in 1236 health promotion practice and research: Strategies for improving public health. 2002;1:40-70. social psychological approach to understanding and promoting health behavior. Social psychological 1239 foundations of health and illness. 2003;22:82-106.

1241 Social Psychological Approach to Understanding and Promoting Health Behavior. 2003:82-106. Jeffrey D.Fisher WAF. <Changing AIDS-Risk Behavior>. Psychological Bulletin. 1992;111(3):455-74. Jones D, Cook R, Rodriguez A, Waldrop-Valverde D. Personal HIV knowledge, appointment 1244 adherence and HIV outcomes. AIDS and behavior. 2013;17(1):242-9. 
1247 Diseases Society of America. 2003;36(4):514-8.

1248 Swindells S, Mohr J, Justis JC, Berman S, Squier C, Wagener MM, et al. Quality of life in patients with human immunodeficiency virus infection: impact of social support, coping style and hopelessness. International journal of STD \& AIDS. 1999;10(6):383-91.

Swahn MH, Braunstein S, Kasirye R. Demographic and Psychosocial Characteristics of Mobile Phone Ownership and Usage among Youth Living in the Slums of Kampala, Uganda. Western Journal of 1253 Emergency Medicine. 2014.

$$
\text { UBOs. Statistical Report } 2018 .
$$
2016;60:243-51. Prototype Mobile App for Health Management for Persons Living with HIV. Studies in health technology 1263 and informatics. 2016;225:481-5. intervention for peer health workers on AIDS care in rural Uganda: a mixed methods evaluation of a cluster-randomized trial. AIDS and behavior. 2011;15(8):1776-84. 
1271 Clinical Outcomes in Low- and Middle-Income Countries for Achieving the UNAIDS 90-90-90 Targets. Curr 1272 HIV/AIDS Rep. 2016;13(5):241-55.

1273

Luxton DD, June JD, Chalker SA. Mobile Health Technologies for Suicide Prevention: Feature

1274 Review and Recommendations for Use in Clinical Care. Current Treatment Options in Psychiatry.

$1275 \quad 2015 ; 2(4): 349-62$.

1276

Amith M, Loubser PG, Chapman J, Zoker KC, Rabelo Ferreira FER. Optimization of an EHR mobile

1277 application using the UFuRT conceptual framework. AMIA Annu Symp Proc. 2012;2012:209-17.

1278 Doris Caliz XA. Usability Evaluation Method for Mobile Applications for the Elderly: A 1279 Methodologica Proposal.

Burnay J, Bushman BJ, Laroi F. Effects of sexualized video games on online sexual harassment.

1281 Aggressive behavior. 2019;45(2):214-23. devices for accessing healthcare information by adolescents. Applied clinical informatics. 2012;3(04):35666.

Sparkes J, Valaitis R, McKibbon A. A usability study of patients setting up a cardiac event loop recorder and BlackBerry gateway for remote monitoring at home. Telemedicine and e-Health. 2012;18(6):484-90. willingness to receive mHealth services among patients with diabetes in Northwest Ethiopia: a crosssectional study. BMJ open. 2019;9(1):e021766-e. 
Version 3.1, Jan 2021

1295

1296

1298

1300

1301

1302

1303

1304

1305

1306

1307

1308

1309

1310

1311

1312

1313

1314

1315

1316

mobile phone-based pharmacy and other health technology services: a pilot study. Telemedicine journal and e-health : the official journal of the American Telemedicine Association. 2014;20(2):182-5.

DeSouza SI, Rashmi MR, Vasanthi AP, Joseph SM, Rodrigues R. Mobile phones: the next step towards healthcare delivery in rural India? PLoS One. 2014;9(8):e104895.

Kim J, Zhang W, Nyonyitono M, Lourenco L, Nanfuka M, Okoboi S, et al. Feasibility and acceptability of mobile phone short message service as a support for patients receiving antiretroviral therapy in rural Uganda: a cross-sectional study. J Int AIDS Soc. 2015;18(1):20311-.

Rana Y, Haberer J, Huang H, Kambugu A, Mukasa B, Thirumurthy H, et al. Short Message Service (SMS)-Based Intervention to Improve Treatment Adherence among HIV-Positive Youth in Uganda: Focus Group Findings. PLoS One. 2015;10(4):e0125187.

Ware NC, Pisarski EE, Tam M, Wyatt MA, Atukunda E, Musiimenta A, et al. The Meanings in the messages: how SMS reminders and real-time adherence monitoring improve antiretroviral therapy adherence in rural Uganda. AIDS (London, England). 2016;30(8):1287-94.

Kunutsor S, Walley J, Katabira E, Muchuro S, Balidawa H, Namagala E, et al. Using Mobile Phones to Improve Clinic Attendance Amongst an Antiretroviral Treatment Cohort in Rural Uganda: A Crosssectional and Prospective Study. AIDS and behavior. 2010;14(6):1347-52.

Siedner MJ, Haberer JE, Bwana MB, Ware NC, Bangsberg DR. High acceptability for cell phone text messages to improve communication of laboratory results with HIV-infected patients in rural Uganda: a cross-sectional survey study. BMC medical informatics and decision making. 2012;12(1):56.

Haberer JE, Kiwanuka J, Nansera D, Muzoora C, Hunt PW, So J, et al. Realtime adherence monitoring of antiretroviral therapy among HIV-infected adults and children in rural Uganda. AIDS (London, England). 2013;27(13):2166-8. 
trial to evaluate the influence of mobile phone reminders on adherence to first line antiretroviral

1319 treatment in South India--the HIVIND study protocol. BMC medical research methodology. 2010;10:25.

Dynamics of HIV-1 Drug Resistance among Treatment-Naive Individuals in Greece: The Added Value of Molecular Epidemiology to Public Health. Genes. 2017;8(11).

1326 adherence to antiretroviral therapy with mobile phone reminders: results from a cohort in South India.

1327 PLoS One. 2012;7(8):e40723.

antiretroviral therapy adherence among adolescents in low- and middle-income countries: A systematic review of the literature. PLoS One. 2018;13(1):e0189770.

1332 technology-based health behaviour change or disease management interventions for health care 1333 consumers: a systematic review. PLoS medicine. 2013;10(1):e1001362.

1335 health care service delivery processes. A systematic review and meta analysis. PLoS medicine. 1336 2013;10(1):e1001363.

Tomlinson M, Rotheram-Borus ea. Scaling up mHealth: where is the evidence? PLoS medicine. 2013;10(2):e1001382. 
1342 and Cost of an mHealth Intervention in Vulnerable People Living With HIV in Vancouver, Canada: 1343 Prospective Study. JMIR mHealth and uHealth. 2018;6(7).

1344 Anglada-Mart H, \#237, nez, Martin-Conde M, Rovira-Illamola M, Sotoca-Momblona JM, et al. An 1345 Interactive Mobile Phone---Website Platform to Facilitate Real-Time Management of Medication in 1346 Chronically ill Patients. J Med Syst. 2017;41(8):1-8. among a national cohort of US veterans. HIV clinical trials. 2009;10(5):299-305. 2011;19(1):12-6.

Fox MP, Rosen S. Patient retention in antiretroviral therapy programs up to three years on treatment in sub-Saharan Africa, 2007-2009: systematic review. Tropical medicine \& international health : TM \& IH. 2010;15 Suppl 1:1-15. of free cotrimoxazole prophylaxis improves clinic retention among antiretroviral therapy-ineligible clients in Kenya. AIDS (London, England). 2011;25(13):1657-61. in Uganda. Poster, internattional Conference. 2011. diseases : an official publication of the Infectious Diseases Society of America. 2011;52(6):793-800. 
to assess retention rates using mobile phone reminders versus physical contact tracing in a potential HIV

1368 vaccine efficacy population of fishing communities around Lake Victoria, Uganda. BMC infectious diseases.

$1369 \quad 2018 ; 18(1): 591$.

1370 Tuller DM, Bangsberg DR, Senkungu J, Ware NC, Emenyonu N, Weiser SD. Transportation costs impede

1371 sustained adherence and access to HAART in a clinic population in southwestern Uganda: a qualitative 1372 study. AIDS and behavior. 2010;14(4):778-84.

1373

Kasigeire S. Socio-Economic Conditions and retention of clients in HIV Care and Treatment at

1374 Kagadi Hospital, Uganda. manuscript. 2014.

1375 Emenyonu N, Muyindike W, Habyarimana J, Pops-Eleches C, Thirumurthy H, Ragland K, et al., 1376 editors. Cash transfers to cover clinic transportation costs improve adherence and retention in care in a 1377 HIV treatment program in rural Uganda. 17th Conference on retroviruses and opportunistic infections; 13782010.

1379 Dillingham R, Ingersoll K, Flickinger TE, Waldman AL, Grabowski M, Laurence C, et al. PositiveLinks: 1380 A Mobile Health Intervention for Retention in HIV Care and Clinical Outcomes with 12-Month Follow-Up. 1381 AIDS patient care and STDs. 2018;32(6):241-50.

Blaya JA, Fraser HS, Holt B. E-health technologies show promise in developing countries. Health 1383 affairs. 2010;29(2):244-51.

WHO. Electronic Health Records Manual For Developing Countries2006.

1386 for primary care, Vol. 2. Thousand Oaks, CA, US: Sage Publications, Inc; 1992. p. 177-93. 
1392 program Ac, editor. 2018.

MoH U. Intergrated Health Care Services Package for HIV Prevention, Treatment and Care

Servicea for Uganda. September 2014.

Amith, M.; Loubser, PG.; Chapman, J.; Zoker, KC.; Rabelo Ferreira, FE. Optimization of an EHR mobile application using the UFuRT conceptual framework.. AMIA Annual Symposium proceedings / AMIA Symposium AMIA Symposium; 2012; p. 209-17.

Luxton DD, Mishkind MC, Crumpton RM, Ayers TD, Mysliwiec V. Usability and feasibility of smartphone

Burnay E, Cruz-Correia R, Jacinto T, Sousa AS, Fonseca J. Challenges of a mobile application for asthma video capabilities for telehealth care in the U.S. military. Telemedicine journal and ehealth : the official journal of the American Telemedicine Association. 2012; 18(6):409-12. [PubMed: and allergic rhinitis patient enablement-interface and synchronization. Telemedicine journal and

Sheehan B, Lee YJ, Rodriguez M, Tiase V, Schnall R. A Comparison of Usability Factors of Four Mobile [PubMed: 23215639] Devices for Accessing Healthcare Information by Adolescents. Applied clinical informatics. 2012; 3(4):356-66. [PubMed: 23227134]

Sparkes J, Valaitis R, McKibbon A. A usability study of patients setting up a cardiac event loop recorder and BlackBerry gateway for remote monitoring at home. Telemedicine journal and ehealth : the official journal of the American Telemedicine Association. 2012; 18(6):484-90. [PubMed: 


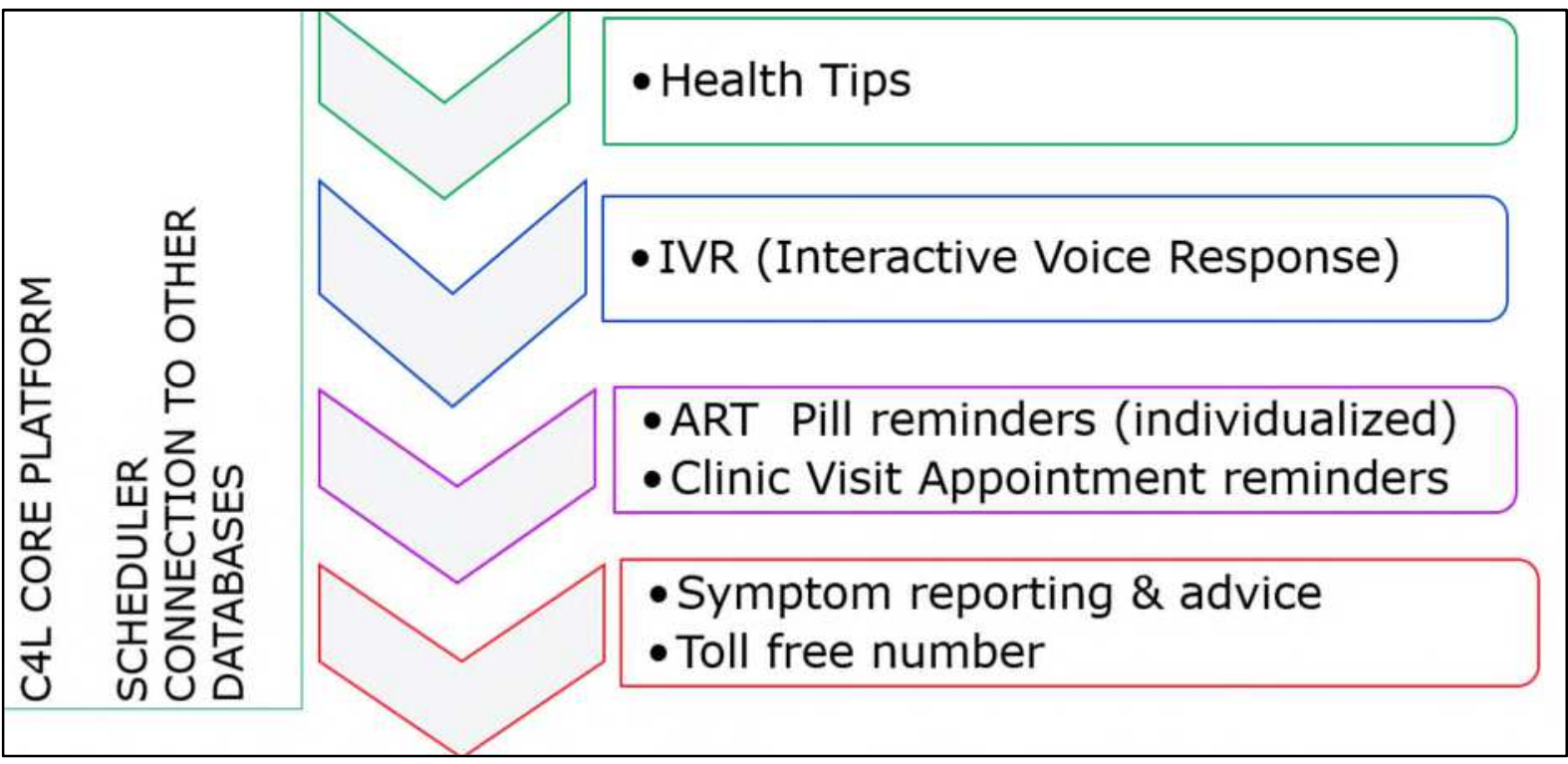

Medicine, Volume XV, NO. 5 : August 2014

H. Anglada-Martinez, G. Riu-Viladoms, M. Martin-Conde, et al. 2015. Does mHealth increase adherence to medication? Results of a systematic review. Int J Clin Pract, January 2015, 69, 1, 9-32. doi: $10.1111 / \mathrm{ijcp} .12$

Figure1: Illustration of Call for life mHealth Tool Functions

Swahn et al, 2014. Mobile Phone Ownership and Usage among Youth. Western Journal of Emergency 

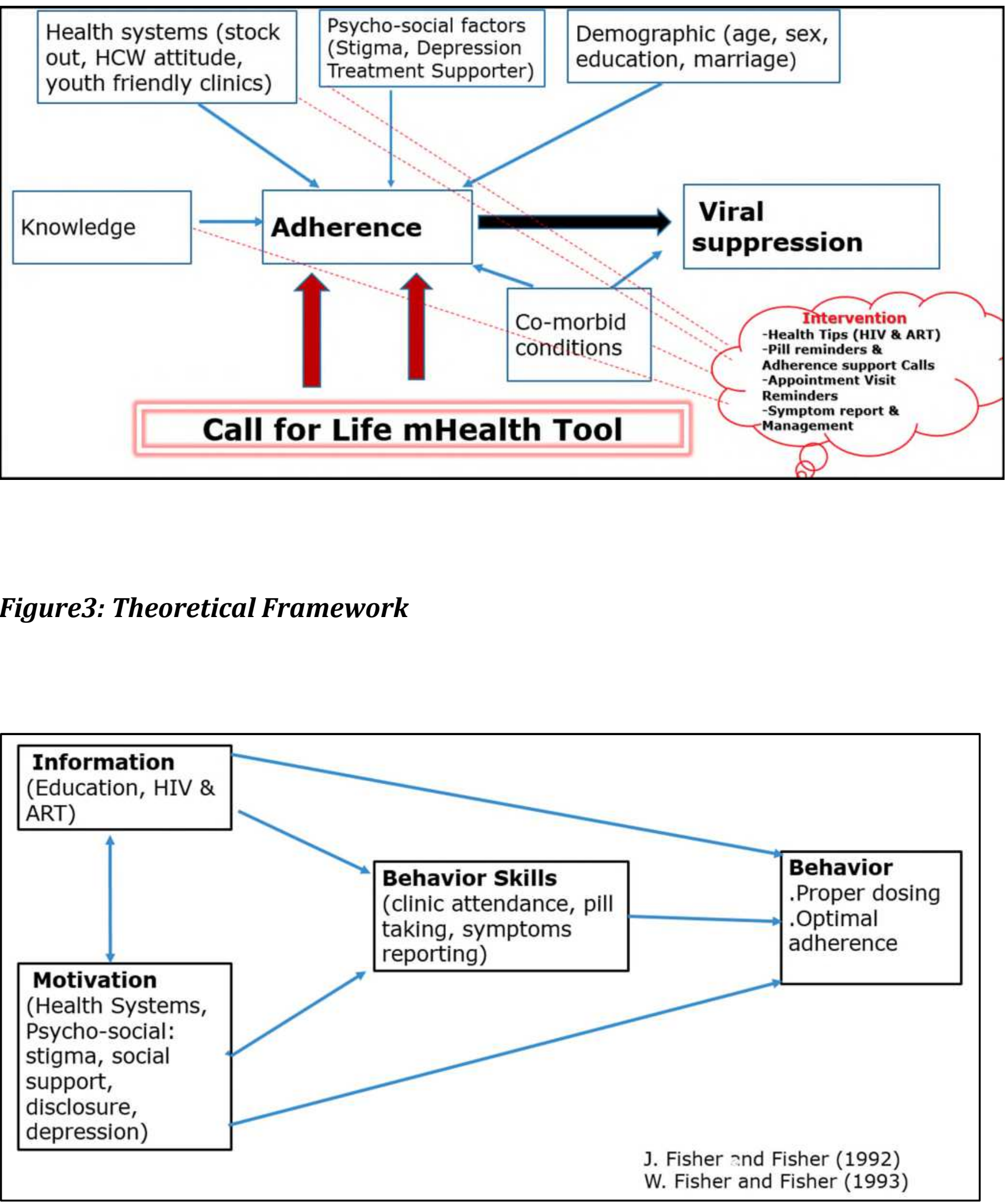
Version 3.1, Jan 2021

Figure 4 Map of Uganda showing Kiryandongo District where study is taking place
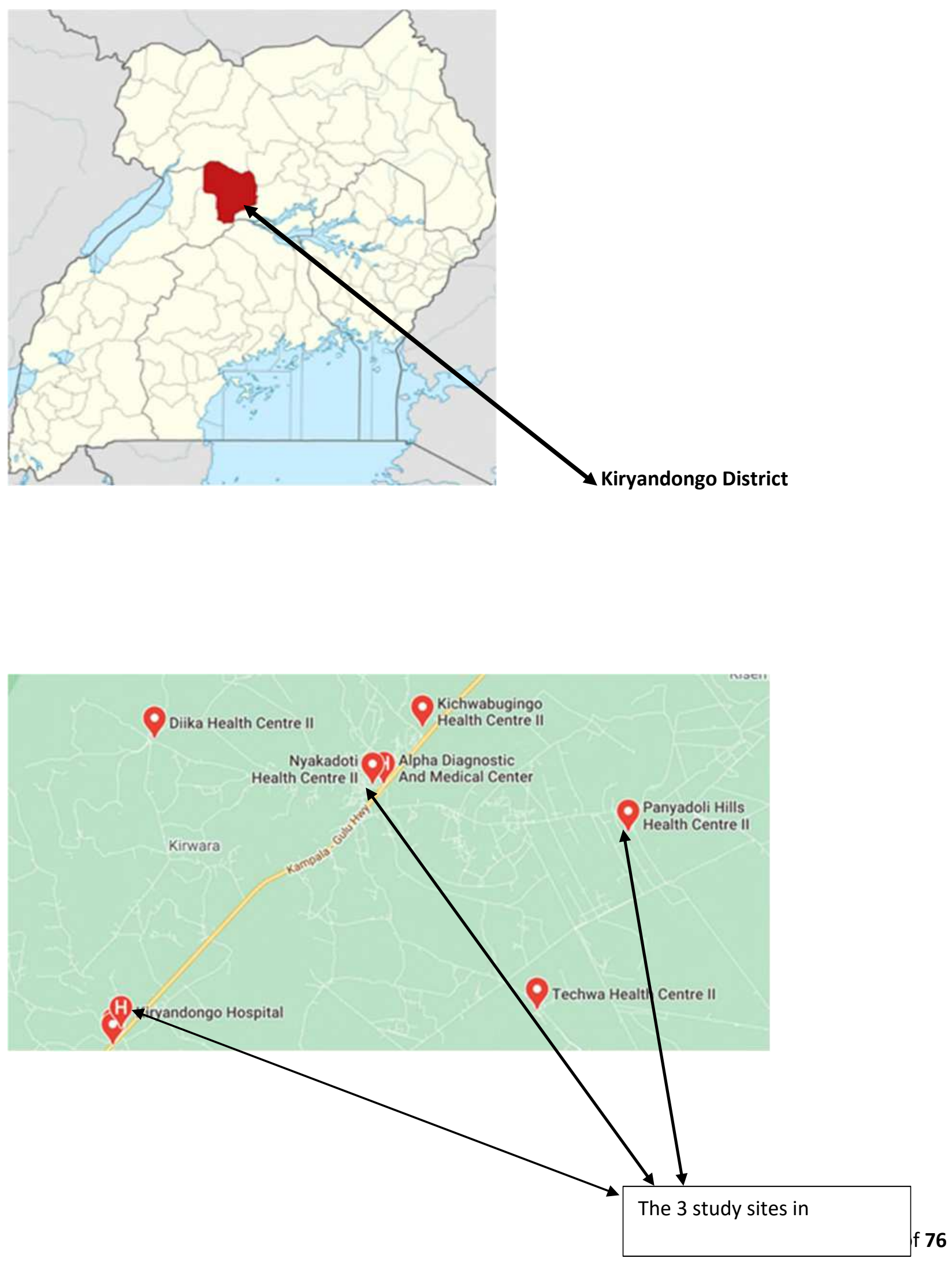


\section{Figure 5: Study Schema}

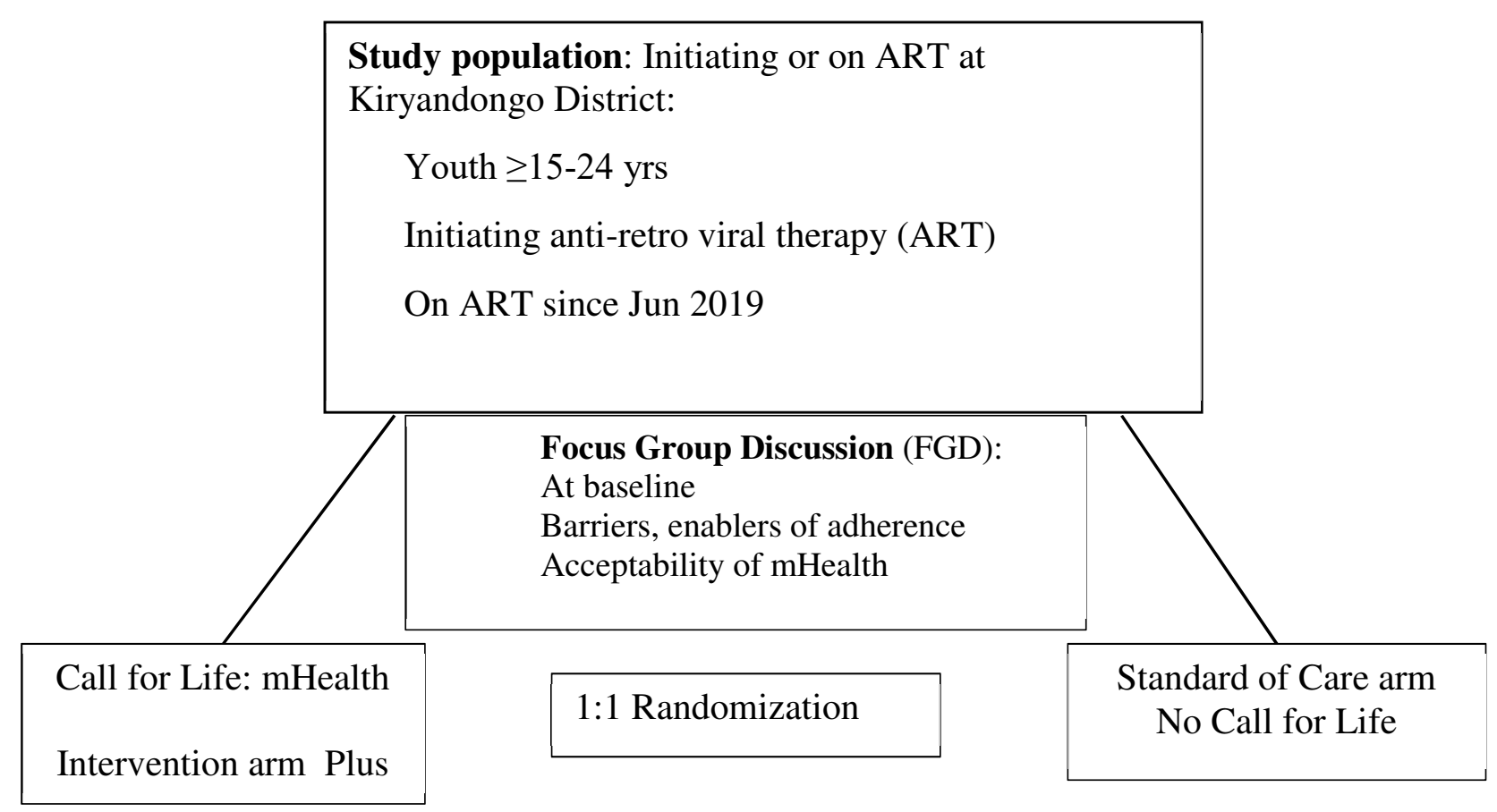

Study questionnaires (Visit $0,1,2$ )

- Socio-demo-medical form

- Stigma scores

- Knowledge assessment

- Sexual behavior

- Knowledge of COVID-19

FGD: At study end

Barriers, enablers of adherence

Utilisation of mHealth 
Outcome assessment at month 6 and 12

ART Adherence; Viral Suppression, Retention in care, Cost-evaluation analysis Knowledge at baseline in comparison to endline 


\section{Supplementary Files}

This is a list of supplementary files associated with this preprint. Click to download.

- Checklist.docx

- EnglishAssentFormYouthStudy.pdf

- EnglishConsentYouthStudy.pdf

- SPIRITchecklist1.docx

- VERSIO3.pdf 